\title{
Experimental confirmation of the IsoRedShift at sunset and sunrise, with consequential absence of the universe expansion and related conjectures ${ }^{1}$
}

\author{
R.M. Santilli*, G. West and G. Amato \\ Magnegas Corporation, Tarpon Springs, FL, USA
}

\begin{abstract}
In this paper, we recall the mutation of the Minkowski spacetime for empty space into the covering isospacetime caused by physical media; we recall its experimental verifications via the isoredshift of laser light in a metal pipe containing air at pressure, as well as additional experimental verifications in other fields; and we present, apparently for the first time, experimental confirmations of Santilli's 1991 prediction of the isoredshift as the primary origin of the redness of direct Sunlight at Sunset and Sunrise without any appreciable relative motion between Earth and Sun, said isoredshift being proportional to the distance traveled by Sunlight in air. Absorption is dismissed as a source of said redness of the Sun because air predominantly absorbs red, as experimentally established, without dismissing minor contributions, e.g., for the shape (but not the shift) of wavelengths. The widely accepted conjecture that scattering is the origin of said redness of the Sun, without dismissing minor contributions, is shown to be inapplicable for direct Sunlight, to have no representational capability for the needed very large shifts (measured up to $200 \mathrm{~nm}$ ), and to have been dismissed experimentally at the cosmological level with recent measurements via Hubble's telescope, as well as on Earth by recent scattering measurements by some of us. Since intergalactic spaces are far from being empty, and physical laws must be the same throughout the universe, the preceding experimental results are shown to be evidence for the absence of the expansion of the universe, the absence of the acceleration of the expansion, the absence of the big bang, and the elimination of the far reaching conjecture that space itself is expanding as needed, from Hubble's law, to prevent a return to the Middle Ages with Earth at the center of the universe. It is finally shown that the same experimental confirmations of Santilli isoredshift imply the absence of dark matter and dark energy since the universe is nowhere empty, with consequential universal validity of Santilli isospacetime and its Lorentz-Poincaré-Santilli isosymmetry.
\end{abstract}

Keywords: IsoRedShift, IsoBlueShift, Doppler shift

\section{Review of preceding measurements of IsoRedShifts}

Following a decade of preparatory mathematical research in the construction of the novel isomathematics with the axiom-preserving Lie-isotopic structure for the invariant treatment of generally nonlinear,

\footnotetext{
${ }^{1}$ This paper is dedicated to Dr. James Gills, M.D., St. Luke's Institute, Tarpon Springs, Florida with appreciation for his invaluable contribution to society, and also contributed to the Proceedings of the San Marino Workshop on Astrophysics and Cosmology for Matter and Antimatter, Republic of San Marino, September 5 to 9, 2011.

${ }^{*}$ Corresponding author: R.M. Santilli, Magnegas Corporation, 150 Rainville Road, Tarpon Springs, FL 23678, USA. E-mail: research@magnegas.com.
}

1472-7978/12/\$27.50 @ 2012 - IOS Press and the authors. All rights reserved

This article is published online with Open Access and distributed under the terms of the Creative Commons Attribution Non-Commercial License 
nonlocal and non-Hamiltonian interior dynamical problems, Santilli [1] presented in 1983 the axiompreserving isotopies of the Minkowski spacetime via the most general possible symmetric (non-singular) spacetime

$$
x^{2}=x_{1}^{1} b_{1}^{2}+x_{2}^{2} b_{2}^{2}+x_{3}^{2} b_{3}^{2}-t^{2} c^{2} b_{4}^{2}=x_{1}^{2} / n_{1}^{2}+x_{2}^{2} / n_{2}^{2}+x_{3}^{2} / n_{3}^{2}-t^{2} c^{2} / n_{4}^{2},
$$

today known as the Minkowski-Santilli isospacetime. In the above covering line element, the terms $b_{\mu}=1 / n_{\mu}$ are called the characteristic quantities of the medium considered (our atmosphere for this paper); they are positive definite functions of all possible local variables, such as distance traveled by light $d$, density $\rho$, temperature $\tau$, light frequency $\nu$, etc., $b_{\mu}=b_{\mu}(d, \rho, \tau, \nu, \ldots)=1 / n_{\mu}=1 / n_{\mu}(d, \rho, \tau, \nu, \ldots)$; they are normalized for the vacuum to the values $b_{\mu}=1 / n_{\mu}=1, \mu=1,2,3,4$; the time characteristic quantity $1 / b_{4}=n_{4}$ is the familiar local index of refraction with corresponding local speed of electromagnetic (e.g., radio) waves $C=c b_{4}=c / n_{4}$; the space characteristic quantities $b_{k}=1 / n_{k}, k=1,2,3$, represent the inhomogeneity and anisotropy of the medium considered; and, rather than being arbitrary parameters as often believed, their value is set by physical evidence (e.g. the value of $1 / b_{4}=n_{4}$ is set by the measure of the speed of the electromagnetic waves within the medium considered, etc.), as we shall see.

As part of the study of isospacetime (1), Santilli constructed in Ref. [1] the axiom preserving isotopies of the Lorentz-Poincare (LP) symmetry and of special relativity (SR), into covering formulations today known as the Lorentz-Poincaré-Santilli (LPS) isosymmetry, and IsoRelativity (IS) (see detailed treatments in monographs [2-4], geometric profiles in Refs [5,6], recent presentation in Ref. [7], general review in Ref. [41] and vast quoted literature).

Besides other predictions experimentally verified elsewhere (see Vol. IV of Refs [4]), the MS isospacetime, the LPS isosymmetry and IR predicted anomalous shifts of the frequency of light propagating within a gaseous medium without any necessary relative motion between the source, the medium and the observer, according to the general isolaw $[1,2 \mathrm{~b}]$

$$
\nu=\nu_{0} /\left(1 \pm \frac{v^{2}}{c^{2}} \frac{b_{3}^{2}}{b_{4}^{2}}+\ldots\right) \approx \nu_{o} /\left(1 \pm \frac{v^{2}}{c^{2}} \frac{n_{4}^{2}}{n_{3}^{2}}+\ldots\right)
$$

holding under a relative motion at speed $v$, today known as the Doppler-Santilli frequency IsoShift, and the particular isoshift

$$
\nu_{v=0}=\nu_{0} /(1 \pm K+\ldots), K=\left[\frac{v^{2}}{c^{2}} \frac{b_{3}^{2}}{b_{4}^{2}}\right]_{v=0}=\left[\frac{v^{2}}{c^{2}} \frac{n_{4}^{2}}{n_{3}^{2}}\right]_{v=0} \neq 0
$$

holding for the case in which the relative motion is null, and known as Santilli IsoShift $[2,3,8]$.

This paper is devoted to measurements without any relative velocity at all according to IsoLaw (3) that can be particularized into: Santilli IsoRedShift (IRS), which occurs for light losing energy $E=h \nu$ to a medium at sufficiently low temperature; IsoBlueShift (IBS), which occurs for light acquiring energy from a medium when at sufficiently high temperature; and NoIsoShift (NIS), which occurs for intermediate conditions.

The above hypothesis were submitted as a necessary background for the main hypothesis of Ref. [2b], namely, that the IRS is the origin of the redness of the Sun at Sunset due to the absence of any relative motion between the Sun, the atmosphere and the observer. Consequently, Santilli submitted in Ref. [2b], page 330 , the following 
Experiment III: Measure in our laboratories the possible isoredshift of sun light in the transition from the zenith to the horizon.

which test is the main objective of this paper.

The most fundamental aspect of these predictions is the hypothesis $[1,2 \mathrm{~b}]$ that energy at large, and matter in particular in any of its possible forms (gas, particles, radiations, etc.) mutate the Minkowski geometry of the vacuum into the covering Minkowski-Santilli isogeometry [5,6] characterized by the most general possible, nonsingular, symmetric spacetime. Under these conditions, the mutation of the characteristics of electromagnetic waves (speed, frequency, polarizations, etc.) is unavoidable. Vice versa, no mutation of any characteristic of electromagnetic waves is possible without a mutation of the underlying spacetime.

Following two decades of rejections by major laboratories around the world to even consider the test of the above predictions, the IRS was first experimentally verified by Santilli $[8,9]$ in 2009 following the construction of the IsoShift Testing Station consisting of: a first air conditioned cabin containing a blue laser light; a second air conditioned cabin containing the wavelength analyzer; and the interconnection of the two cabins via a $60 \mathrm{ft} \approx 18 \mathrm{~m}$ long steel pipe containing air at pressure up to $2,000 \mathrm{psi} \approx$ 1137 bars. Refs $[7,8]$ presented systematic IRS detection for the blue laser light exiting from said pipe in the transition from atmospheric pressure to $2,000 \mathrm{psi} \approx 1137$ bars. Refs [8,9] also presented systematic experimental evidence of the increase of the IRS with the increase of the pressure, that is, with the increase of the travel of light in our atmosphere.

In 2011, West and Amato [10] provided systematic experimental confirmations of the existence of Santilli IRS, as well as the first experimental evidence on the existence of the IBS and NIS via the use of the same IsoShift Testing Station used for measurements [8,9]. West and Amato also provided experimental evidence on the absence of frequency shift for the scattering of light in a gas, thus providing an experimental confirmation on Earth of the parallel dismissal of scattering as the origin of the cosmological redshift of galaxies obtained via the Hubble telescope 9 see, e.g., Ref. [11].

These measurements were then used in Ref. [10] to confirm Santilli's 1991 hypothesis [2b] that the large differences in cosmological redshift between quasars and associated galaxies (when physically connected according to gamma spectroscopic evidence) originate from the IRS within the largely different quasar chromospheres and innergalactic media.

In this paper, we present, apparently for the first time, experimental confirmation of Santilli 1991 hypothesis [2b] that the redness of the Sun at Sunset is caused by the IRS of Sunlight propagating within our atmosphere without any relative motion between the source, the medium and the observer. We also present experimental that said IRS of Sunlight increases with the increase of the travel of light in our atmosphere, that is, with the decrease of the elevation of the Sun on the horizon, as established by visual evidence at Sunset.

Recall that physical laws are expected to apply throughout the universe, that is, from our Earthly environment to the most distant galaxies. Recall also that intergalactic space is far from, being empty, since it consists of a physical medium with a very high energy density originating from light emitted by galaxies throughout the universe, gases, dust, radiation, etc. Consequently, in this paper we assume the preceding evidence on the IRS as experimental evidence obtained on Earth of the main prediction of Refs [8,9], namely, the absence of: the expansion of the universe, the absence of the acceleration of the expansion, the absence of the big bang, the absence of dark matter and energy, and the absence of the the far reaching conjecture that space itself is expanding, an expansion is needed to prevent a return to the Middle Ages with Earth at the center of the universe from Hubble's law. 


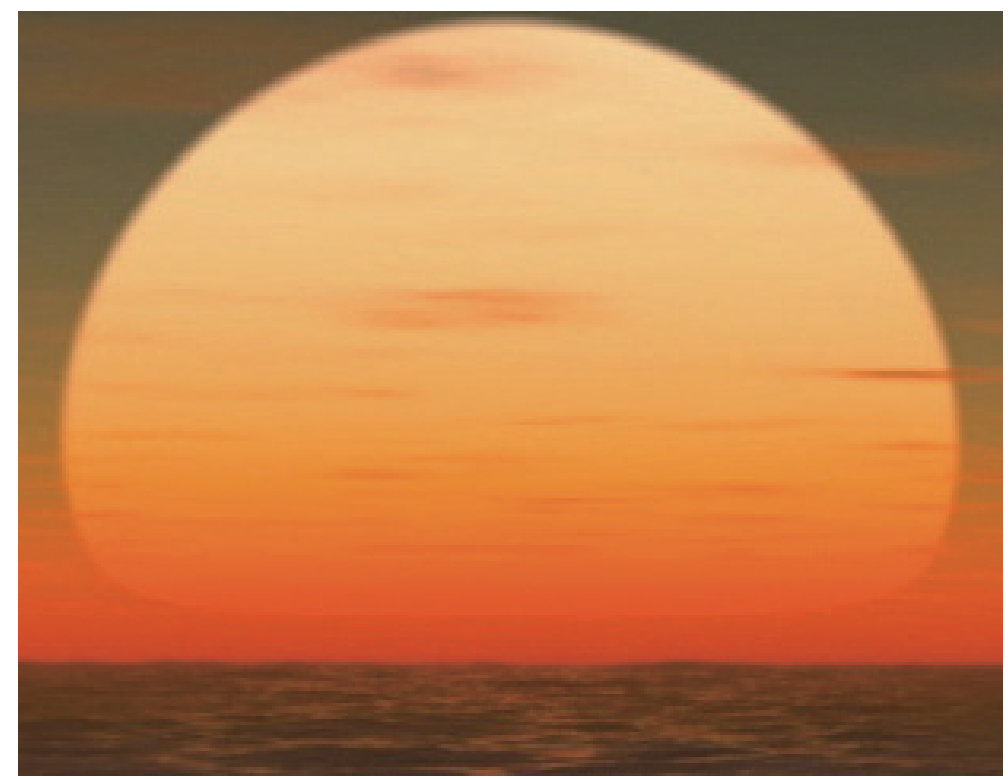

Fig. 1. In this paper we present experimental verifications of the hypothesis submitted by Santilli in Ref. [2b] of 1991, page 330, according to which the redness of "direct" Sunlight at Sunset is due to the IsoRedShift (IRS) based on the loss of energy $E=h \nu$ by light to air at low temperature without any relative motion between the source, the air and the observer. By remembering that physical law have to be the same throughout the universe, the visual evidence of this picture and its experimental confirmation presented in this paper are assumed as evidence on the absence of the universe expansion, with consequential absence of the expansion of space itself and the big bang. The increase of the redness of the Sun at Sunset with the increase of the travel of Sunlight in air visible to the naked eye, and its experimental confirmation presented in this paper, are assumed as evidence on the absence of the acceleration of the expansion of the universe with the increase of the distance from Earth. The same experimental results are then shown to be evidence for the absence of dark matter and dark energy.

The understanding of this paper requires a technical knowledge of the content of at least monograph [2b] and of experimental papers [7-9] we cannot possibly review for brevity. It should be stressed that the main scope of this paper is to establish the IRS "origin" of the redness of the Sun at Sunset and Sunrise, and not the accurate measurement of its numerical value for a given elevation. Needless to say, independent measurements are warmly encouraged, first of all, on the absence of frequency shift in the scattering of light in a gas, and then on the sole remaining possibility for frequency shift for light propagating in a gas, the IRS.

\section{Experimental confirmation of the IsoRedShift origin of the redness of the sun at sunset and sunrise}

\subsection{Statement of the problem}

As it is well known, Sunlight at the Zenith is predominantly yellow but at Sunset is predominantly red, with a redness that increases with the increase of the distance traveled by light in our atmosphere, that is, with the decrease of the elevation of the Sun over the horizon without any appreciable relative motion between the Earth and the Sun, as illustrated in Fig. 1. During Sunrise we have the inverse transition from the redness of the Sun at the horizon to a yellow color at the Zenith also without any appreciable relative motion between the Earth and the Sun. The transition from yellow to red implies an anomalous shift 
of about $55 \mathrm{~nm} /$. However, by recalling that only blue light can reach us at the horizon, the anomalous redshift for both Sunset and Sunrise is expected as being of the order of $100 \mathrm{~nm}$. Bigger values of the anomalous redshift are then expected (up to $200 \mathrm{~nm}$ ) by inspecting the event for infrared frequencies.

Consequently, the problem addressed in this section is to achieve an experimental verification of the representation of the redshift of the Sun in the transition from the Zenith to Sunset which is quantitative, invariant over time and increases with the increase of the travel of Sunlight in our atmosphere. The results will then be applied for the decrease of the redness of the Sun in the transition from Sunrise to the Zenith.

In the above statement of the problem, the term "quantitative" is referred to a numerical representation of the increase of the redness with the increase of the travel of Sunlight in our atmosphere; the numerical representation of the entire indicated shift up to values of the order of $100 \mathrm{~nm}$; and the condition of "invariance over time" is referred to the condition that the selected representation must predict the same numerical values under the same conditions at different times [1-9].

The authors would like to stress that the aim of this paper is to provide experimental evidence on the primary IRS "origin" of the redness of the Sun at the horizon, with the clear understanding that the achievement of its "exact" numerical value will likely require decades of tests.

\subsection{Possible representations}

The redness of the Sun at Sunset and Sunrise has been observed by mankind since the dawn of civilization, and its scientific study has been conducted since Galileo's time. During this long period of time, numerous hypotheses have been formulated on the origin of the indicated redness, the most important ones deserving consideration being nowadays reduced to three: absorption, scattering and IsoRedShift.

\subsection{Inapplicability of special relativity within our atmosphere}

Contrary to the case of galaxies, in the case of the Sun we have visual evidence of the absence of an appreciable relative motion between Sun and Earth during the short duration of the Sunset. Consequently, the redness of the Sun at Sunset constitutes visual evidence of the violation of the Doppler shift law in our atmosphere, with consequential inapplicability of special relativity, as it is the case at large within all physical media [1-9].

Independently from this visual evidence, there are numerous additional reasons according to which special relativity cannot possibly be exactly valid in our atmosphere, such as:

1) Our atmosphere is inhomogeneous (because of the variation of the density with the elevation from the sea level) and anisotropic (because of the rotation of Earth). These features imply the collapse of the geometric foundations of special relativity, the Minkowskian spacetime, since the latter strictly requires homogeneity and isotropy;

2) Dynamics in our atmosphere, such as a satellite during re-entry, requires contact, zero range, nonpotential interactions (e.g., of resistive type) that are beyond any hope of representation via a Lagrangian or a Hamiltonian, thus being beyond the analytic foundations of special relativity, since the latter solely admits the action-at-a-distance, potential interactions entirely represented by a Lagrangian or a Hamiltonian. Additionally, it should be mentioned, for readers without technical knowledge of the field, that the reduction of macroscopic objects moving in our atmosphere to their elementary particle constituents (for the evident purpose of regaining special relativity at the particle level), has been disqualified as being inconsistent by the No reduction Theorems (see, e.g., Vol. I of Refs [5]). 
3) As it is well known, special relativity can only be defined with respect to inertial reference systems, that is, reference frame moving in empty space along a straight line without acceleration. Evidently, such inertial frames cannot exist in our atmosphere, with consequential impossibility of properly formulating the basic axioms of special relativity, let alone verifying them experimentally.

4) The strict application of special relativity within our atmosphere implies the violation of causality. In fact, particles such as cosmic rays can travel in our atmosphere at speeds bigger than the local speed of electromagnetic (e.g., radio) waves, thus implying a violation of causality. For non-expert readers in the field, it should be recalled that the reduction to photons of all electromagnetic waves propagating within our atmosphere has been dismissed as being inconsistent on numerous grounds (inability to provide a numerical representation of the angle of refraction, the reduction of speed, the propagation of a light beam along a straight line, etc.), without denying the existence of quantized absorptions, but evidently only for certain frequencies characterized by the atomic structure of our atmosphere [8].

5) When the speed of light in vacuum is assumed as the maximal causal speed within our atmosphere (for the evident attempt of regaining causality), we have the violation of the relativistic laws of addition of speeds because the sum of the speeds of two electromagnetic (again, e.g., radio) waves in our atmosphere does not yield the local speed of electromagnetic waves; and other reasons [1-9].

Despite the above diversified evidence on the inapplicability of special relativity in our atmosphere, the absorption and scattering hypotheses have been formulated as the primary origin of the redness of the Sun at Sunset and Sunrise for the generally unspoken, but specific intent of adapting physical evidence to preferred Einsteinian theories and, because of said intent, the hypotheses have acquired a rather vast acceptance in the physics community.

\subsection{Dismissal of the absorption hypothesis}

The hypothesis that absorption is the origin of the redshift of the Sun at Sunset and Sunrise has no scientific foundations. Visual evidence establishes beyond credible doubt that the sky is blue because red light is absorbed by our atmosphere following a maximal effective vertical travel of about $49 \mathrm{mi} \approx 80 \mathrm{~km}$.

The trajectory of Sunlight at Sunset or Sunrise, that is, the trajectory along a tangent to Earth at the horizon, is of of the order of $4356 \mathrm{mi} \approx 7000 \mathrm{~km}$ (given by Earth's radius of 3,968 $\mathrm{mi} \approx 6,384 \mathrm{~km}$ plus the atmosphere along said tangent). Therefore, the trajectory of Sunlight at the horizon is about 875 times bigger than that for the Sun at the Zenith.

It is then evident that, since red light cannot reach us when the Sun is at the Zenith, the same red light cannot possibly reach us when Sunlight covers a trajectory 875 times longer than that at the Zenith.

Independently from the above evidence, had the Sun been dark blue at Sunset or Sunrise, then the absorption conjecture would be scientifically valid. But the Sun is red at Sunset and Sunrise, thus providing visual evidence that the absorption hypothesis here considered is essentially a non-scientific attempt to adapt physical reality to the preferred Einsteinian theories.

Almost needless to say, the evidence that our atmosphere absorbs light is equally incontrovertible, as discussed in more details below. The aspect here addressed is that the absorption of individual spectral lines by our atmosphere positively cannot cause any frequency shift because said absorption is set by the atomic structure of our atmosphere. Therefore, the sole possibility for a frequency shift due to absorption is that of changing the chemical composition of our entire atmosphere. 

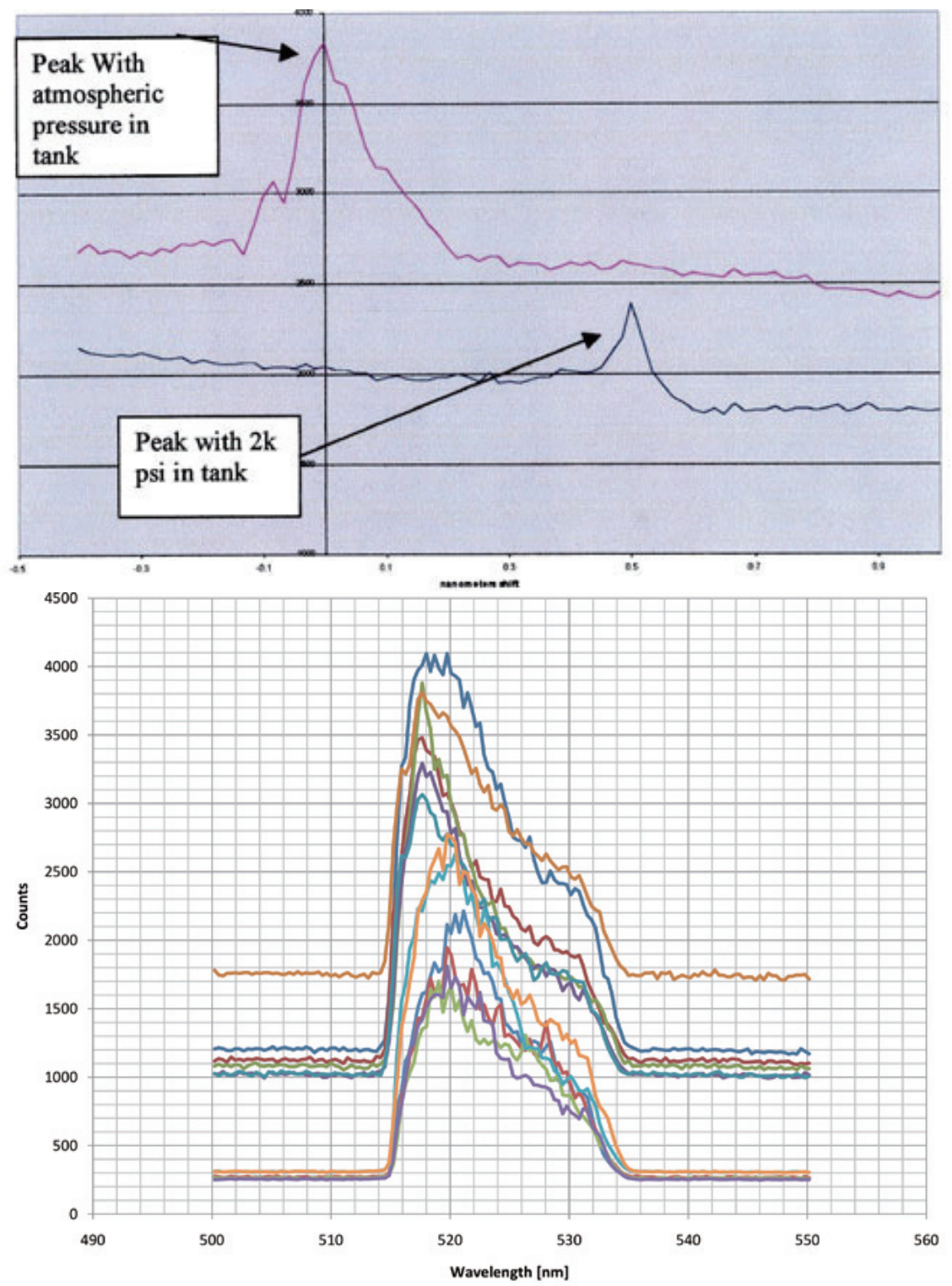

Fig. 2. A view of the first experimental confirmations of Santilli 1991 hypothesis of the IRS in air achieved in Ref. [8] of 2009 for a monochromatic laser light in a pressure tube. The top picture shows the first IRS measurement at pressure, while the bottom picture shows its increase with the increase of the air pressure or, equivalently, of the travel of the laser light in air. Independent comprehensive confirmations were then provided in Ref. [10].

\subsection{Dismissal of the scattering hypothesis}

To begin, it should be stressed that the conjecture on the scattering origin of the redness of the Sun at Sunset and Sunrise (see e.g., Refs [12-18]) is purely theoretical without any experimental verification, whether direct or indirect. As a matter of fact, said scattering origin has been dismissed experimentally by West and Amato [10] as well as by astrophysical measurements obtained via the Hubble telescope [11]. In any case, by remembering that intergalactic spaces are far from being empty, and that physical laws 


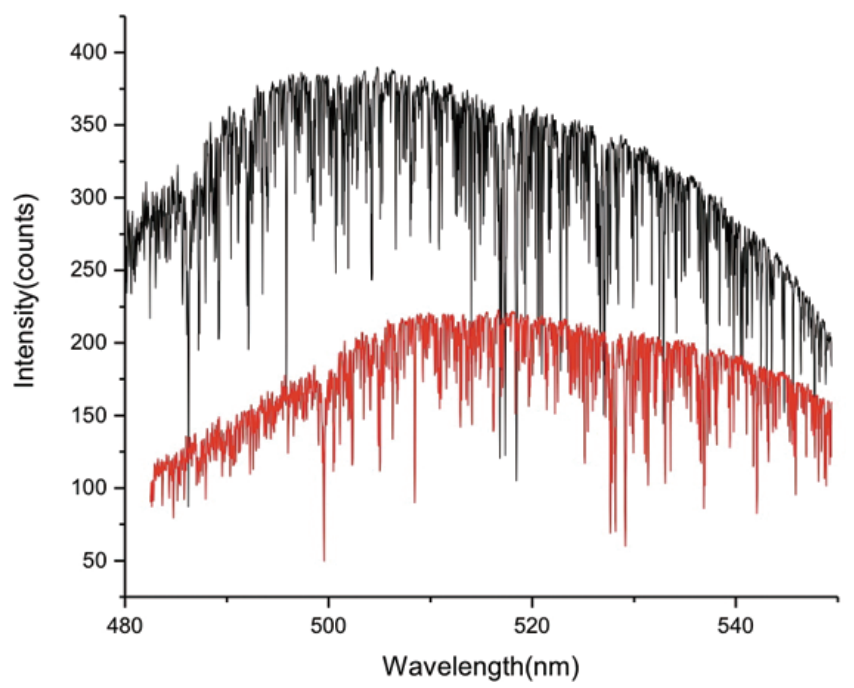

Fig. 3. An electronically created composition to illustrate the definition of the IRS for the spectrum of Sunlight, rather than that for monochromatic wavelengths as in Fig. 2, showing: 1) The spectrum of actual Sunlight at the Zenith (top); 2) The same spectrum electronically shifted by $47 \mathrm{~nm}$ (bottom); and 3) Both spectra being cut from 480 to $580 \mathrm{~nm}$. The main feature illustrated in this picture is that a visual comparison of the two spectrograph shows no apparent frequency shift, while in reality we have a shift of about $47 \mathrm{~nm}$. This illustrates the impossibility to identify the IRS of individual wavelengths when dealing with a spectrum, and the consequential need to measure the IRS of the entire spectrum pf Sunlight in the transition from the Zenith to the horizon.

must be the same throughout the universe, supporters of the scattering origin of the redness of the Sun at Sunset and Sunrise cannot credibly dismiss the scattering of light in intergalactic gases as the origin of the cosmological redshift while jointly assuming the opposite view for the Sun. To put it explicitly, supporters of the scattering hypothesis here considered must dismiss the expansion of the universe on sole grounds of scientific coherence and universality of physical laws.

It should be recalled that Santilli [1-9] rejected scattering as the origin of the redness of the Sun at Sunset and Sunrise on grounds very similar to those used for the rejection of scattering as the origin of the cosmological redshift of galaxies, that is, scattering cannot possibly occur along a straight line for all frequencies, scattering cannot possibly generate a redshift of the entire Solar spectrum, and for other reasons, thus warranting the conduction of Experiment III [2b] reviewed in Section 1.

Needless to say, the following physical evidence should be recalled to prevent misinterpretations:

1) The color of our atmosphere is precisely due to scattering because, in its absence, our sky would be black day and night;

2) Scattering of some of direct Sunlight in our atmosphere is the origin of the loss of intensity of Sunlight;

3) The color of our atmosphere surrounding the Sun at Sunset or Sunrise is due precisely to scattering of red light;

4) A component of the redness of the atmosphere surrounding the Sun at Sunset does indeed contribute to the detected redness of the Sun at Sunset and Sunrise in our instruments, such as a telescope (Fig. 4). However, simple measurements establish that the latter contribution is so small compared to the redness of "direct" Sunlight to be ignorable in first approximation, as done in this paper; and, finally, 


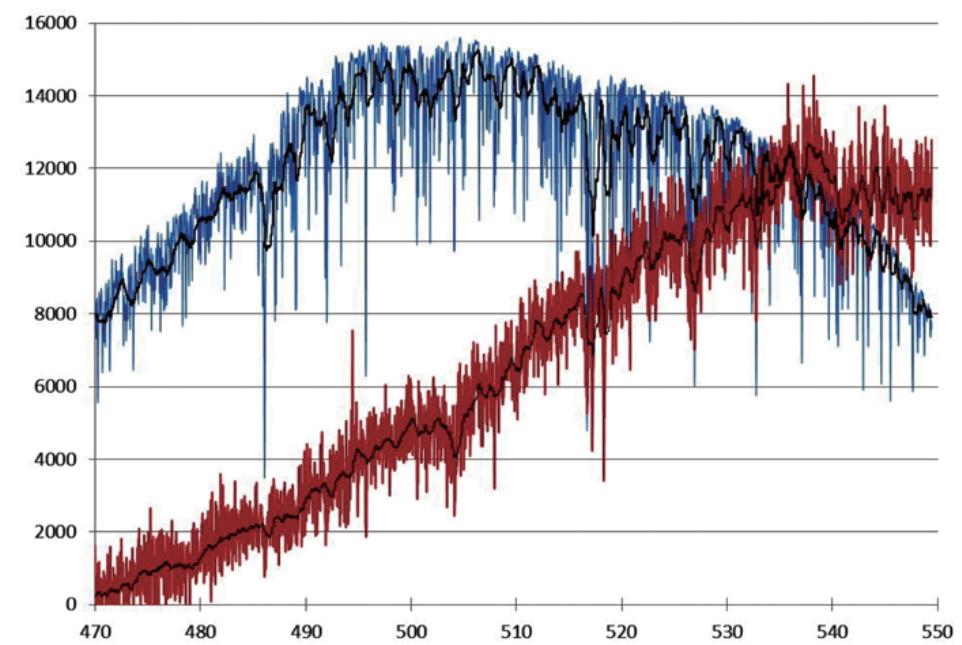

Fig. 4. The view of the spectrograms of direct Sunlight at the Zenith (top) and at the horizon (bottom) obtained on July 19, 2011, with the Avantes analyzer model 3648-FCPC and cut from 470 to $550 \mathrm{~nm}$. As one can see, the comparison of the two spectrograms shows no apparent IRS, except for an irrelevant change in intensity. This occurrence is fully in line with the definition of large IRS for a spectrum (rather than for a monochromatic wavelength) because a shifted segment of a spectrum must essentially coincide with the original spectrum segment. Hence, the use of the Avantes analyzer model 3648-FCPC, while being excellent for the detection of the IRS of monochromatic wavelengths as in Ref. [10], was inconclusive for the measurement of the IRS of the Sun at Sunset because its range from 470 to $550 \mathrm{~nm}$ was insufficient to cover the entire spectrum of direct visible Sunlight.

5) 2) Our atmosphere is blue during the day and red at the horizon at Sunset and Sunrise, thus establishing that scattering occurs for all frequencies and cannot possibly be the origin of the shift from, blue to red.

\subsection{Experimental confirmation of the IRS hypothesis}

Let us begin by identifying the main aspect of the IRS ot the Sun at Sunset we intend to verify experimentally. As established in Refs [8-10], for the case of the propagation of a monochromatic wavelength through air, the IRS is clearly detectable without ambiguities, as shown since the original IRS scan of June 12, 2009 (Fig. 2).

For the case of the Sun at Sunset and Sunrise the situation is quite different because the IRS hypothesis predicts that the entire spectrum of Sunlight is shifted for about $55 \mathrm{~nm}$ from yellow $570590 \mathrm{~nm}$ at the Zenith to red $620750 \mathrm{~nm}$ at the horizon due to the loss of energy $E=h \nu$ by all frequencies to the traversed atmosphere, along essentially the same occurrence as that for the cosmological redshift. In particular, the IRS hypothesis holds instead of the IBS because the atmosphere is predominantly at low temperature soon after leaving the sea level, as well known.

In order to illustrate the main feature, we present in Fig. 3 an electronically created IRS showing, at the top, the spectrum of the Sun at the Zenith and, at the bottom, the same spectrum electronically shifted by about $47 \mathrm{~nm}$. The main point illustrated in Fig. 3 is that, when both spectra are cut at the same width from 450 to $570 \mathrm{~nm}$, there is no clear frequency shift at all, while in reality we have an electronic IRS of about $47 \mathrm{~nm}$.

Consequently, the main difference between monochromatic laser lights and the spectrum of the Sun is that the IRS can be well identified for the former [8-10], while for the latter cannot any longer be 


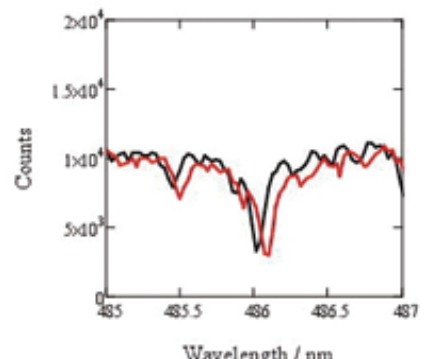

Wavelength $/ \mathrm{nm}$

- REDSHIFT010008

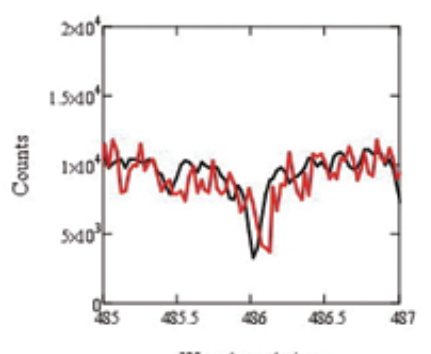

Wavelength $/ \mathrm{nm}$

- REDSHIFT 010008
- REDSHIFT 010020

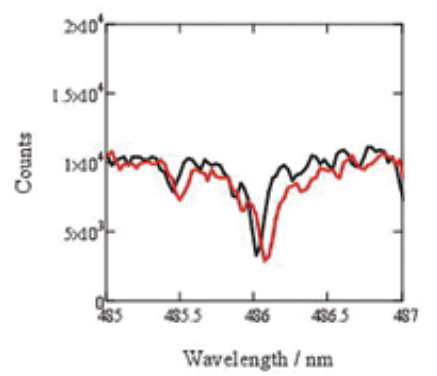

- REDSHIFT010008

- REDSHIFT010014

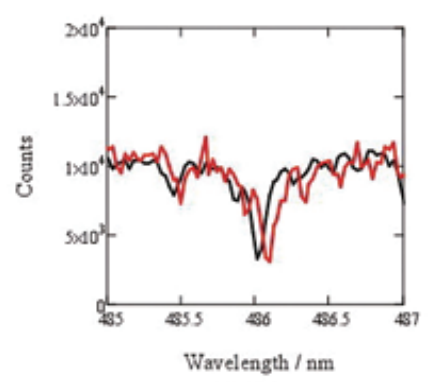

- REDSHIFT010008

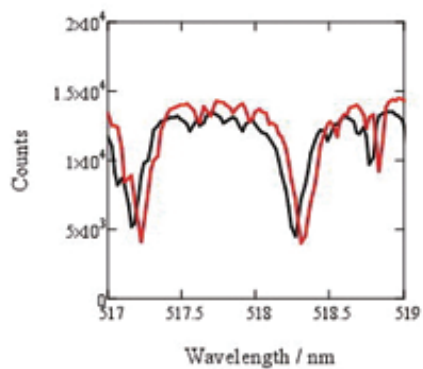

Wavelength $/ \mathrm{nm}$

— REDSHIFT 010008

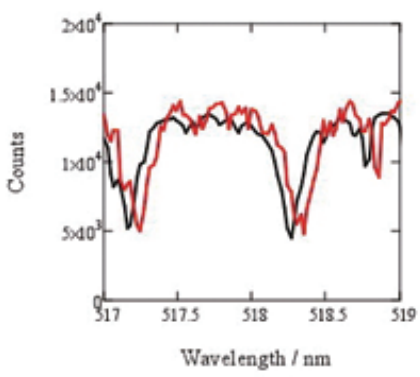

- REDSHIFT010008

- REDSHIFT 010020

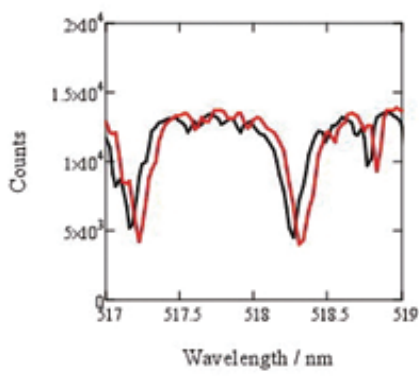

- REDSHIFT010008

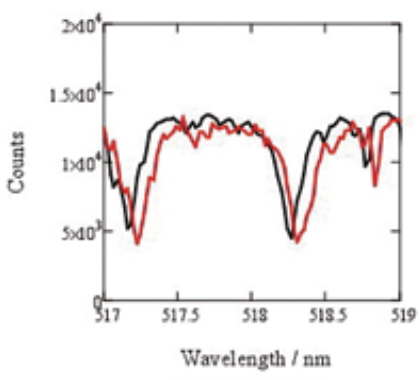

- REDSHIFT010008

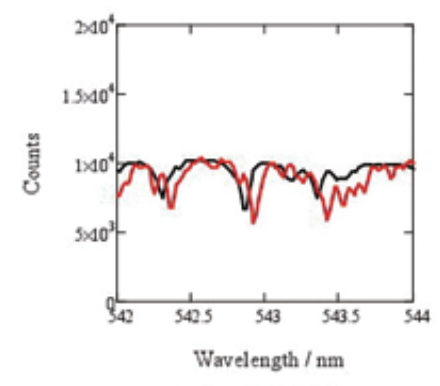

— PEDSHIFT010008

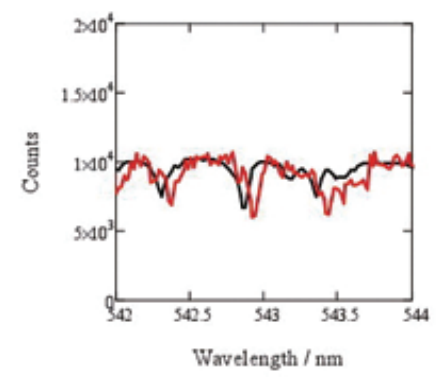

- REDSHIFT010008

- REDSHIFT 010020

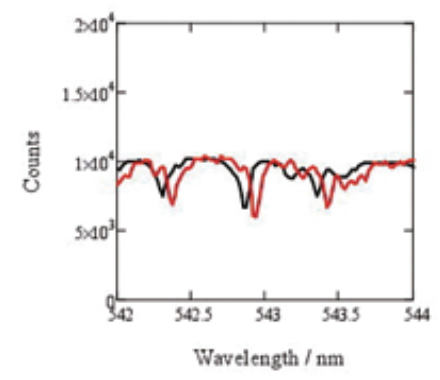

- REDSHIFT010008

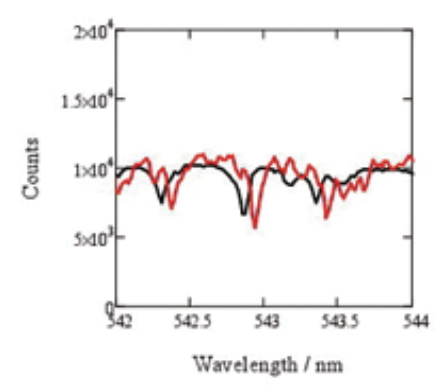

- REDSHIFT010008

Fig. 5. Spectrograms of representative wavelengths of the Sun at the Zenith (left) and at about $60^{\circ}$ elevation on the horizon (right) establishing an IRS of the order of $0.06 \mathrm{~nm}$. The significance of these measurements is that, at said elevation, no redness of the Sun is visible to the naked eye. Nevertheless, spectrographic analysis shows the existence of systematic, IRS. With the decrease of the elevation, the IRS of individual wavelengths increases to such values (of the order of $55 \mathrm{~nm}$ and more) not to be detectable, thus leaving as the sole alternative the measurements of the IRS of the entire spectrum of the direct visible Sunlight. 


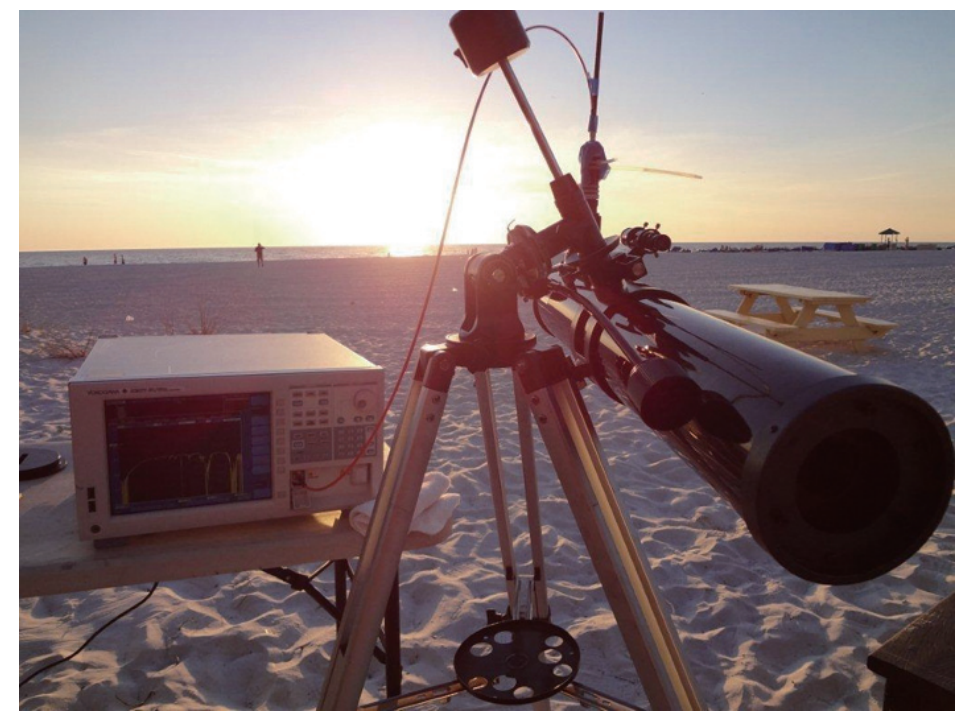

Fig. 6. View of the experimental set up used for the measurement of the IRS of the entire visible spectrum of Sunlight showing the telescope and the Yokogawa analyzer AQ6373 set for the detection at the horizon.

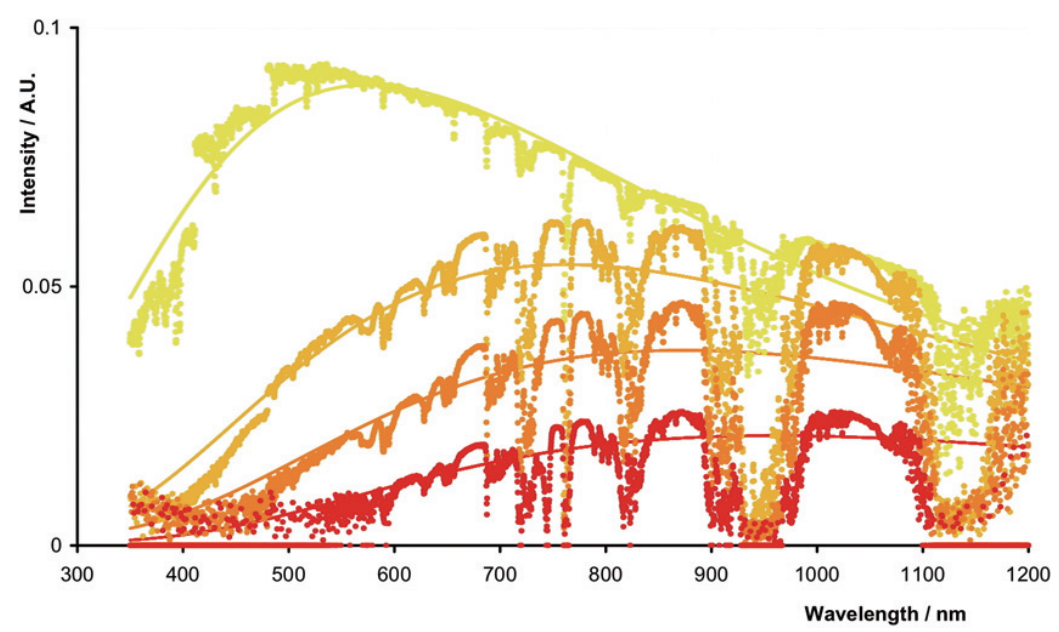

Fig. 7. Chromatographic analysis of the entire spectrum of direct Sunlight obtained on March 16-17, 2012, with the Yokogawa analyzer AQ6373 referred to from the top down the Zenith, $3^{\circ}, 1^{\circ}$, and $0^{\circ}$ degrees elevations from the horizon providing clear evidence of the IRS as the primary origin of the redness of the Sun at Sunset. Due to the size of the IRS (estimated to be of the order of $55 \mathrm{~nm}$ ), absorption and scattering contributions are ignorable in first approximation (comprehensive spectrograms, pictures and other information are available in Ref. [19]).

identified at the level of individual wavelengths, thus leaving as the only possible detection the scan of the entire direct visible Sunlight from the Zenith to the horizon.

Due to the lack in 2009 of the necessary instruments for the measurement of direct Sunlight, Santilli [8, 9] constructed the IsoShift Testing Station and established the existence of the IRS in air at pressure in the pipe Fig. 2) as a preliminary evidence specifically intended for the expected IRS origin of the redness of the Sun at Sunset and Sunrise.

Thanks to the recent availability of the necessary instruments, from May 2011 to early 2012 the 
authors conducted measurements of "direct" visible Sunlight with the use of a commercially available telescope condensing Sunlight directly into the wavelength analyzer by Avantes, model Avaspec-3648USB2-FCPC capable of detecting frequencies from 450 to $570 \mathrm{~nm}$ (from green to red).

Figure 4 presents the comparison of direct Sunlight at the Zenith and at the horizon cut to the range from from 450 to $570 \mathrm{~nm}$. As one can see, the comparison shows no apparent IRS, exactly as predicted (see Fig. 3). However, this does not necessarily mean lack of IRS in the needed large value due to the predicted identity of the original and isoshifted spectrum, except for different intensity and other minor differences.

Nevertheless, we detected a small IRS of the order of $0.06 \mathrm{~nm}$ when the Sun was at about $70^{\circ}$ over the horizon, as reported in Fig. 5. Despite its small character due to the high elevation, these measurements are significant because they establish the existence of the IRS in Sunlight even when no redshift is visible to the naked eye.

When reaching the limit of measurable Sunlight at Sunset permitted by our instrument which is an elevation of the order of $2^{\circ}-3^{\circ}$ at the center of the Sun disc (rather than at its bottom where the biggest redshift occurs). the IRS became too large for clear identification wavelength per wavelength.

We should mote again that absorption lines cannot experience frequency shift because absorption is characterized by the molecules of the atmosphere. Therefore, the only way to have a frequency shift from absorption lines would be to change the chemical composition of our atmosphere.

Note that the setting of Fig. 4 is similar to that for cosmological redshifts. In fact, we can assume the spectrum of light from a nearby galaxy with a small redshift, and the spectrum from a faraway galaxy with a large redshift. Then, both spectra from 450 to $550 \mathrm{~nm}$ have the same frequencies, and one would be tempted to conclude that there is no cosmological redshift contrary to experimental evidence.

In view of the above insufficiencies, we secured the Yokogawa analyzer model AQ6373 with the range from 400 to $1100 \mathrm{~nm}$ and conducted systematic measurements of direct visible Sunlight from the Zenith down to $0^{\circ}$ degree elevation with the set up of Fig. 6. In Fig. 7, we report a chromatographic analysis of all scans that established indeed an IRS of the order of $55 \mathrm{~nm}$ for the transition from yellow to red, plus additional IRS beyond red. As a consequence of such a large values of the IRS, contributions from absorption and scattering can be indeed ignored in first approximation.

In Fig. 8, we present again small values of the IRS for large elevation. In Fig. 9, we present the chromatographic analysis of the scans for the maximal wavelengths from the Zenith to the horizon, confirming the large value of the IRS which is of the order of $55 \mathrm{~nm}$ when referred to the transition from yellow to red, but of $200 \mathrm{~nm}$ or more when referred to the transition from, blue to infrared.

In Fig. 10, we present the analysis of the chromatograms here considere-establishing the increase of the IRS with the increase of the travel of Sunlight in air achieved via an increase of the density for the same distance. Figure 11 presents some of the original spectrograms obtained via the Yokogawa analyzer AQ6474, The complete series of the original scans, pictures and other data are available for free download from the link of Ref. [19].

In addition to the above experimental evidence of the IRS origin of the redness of the Sun at Sunset (see below for Sunrise), no mature judgment can be reached without the consideration of the following indirect evidence:

1. The IRS established for the pipe with air at pressure [8-10] constitutes experimental evidence for the IRS of the Sun at Sunset because the medium (our atmosphere) is the same in both cases. The statement then follows from the universality of physical laws. As a matter of fact, the IRS in the pipe is such to allow a numerical prediction of the IRS at Sunset [8]. Alternatively, any dismissal of the IRS for the Sun at Sunset, to achieve credibility, must prove its compatibility with the existence of the IRS in the pipe with air at pressure, which is clearly impossible. 


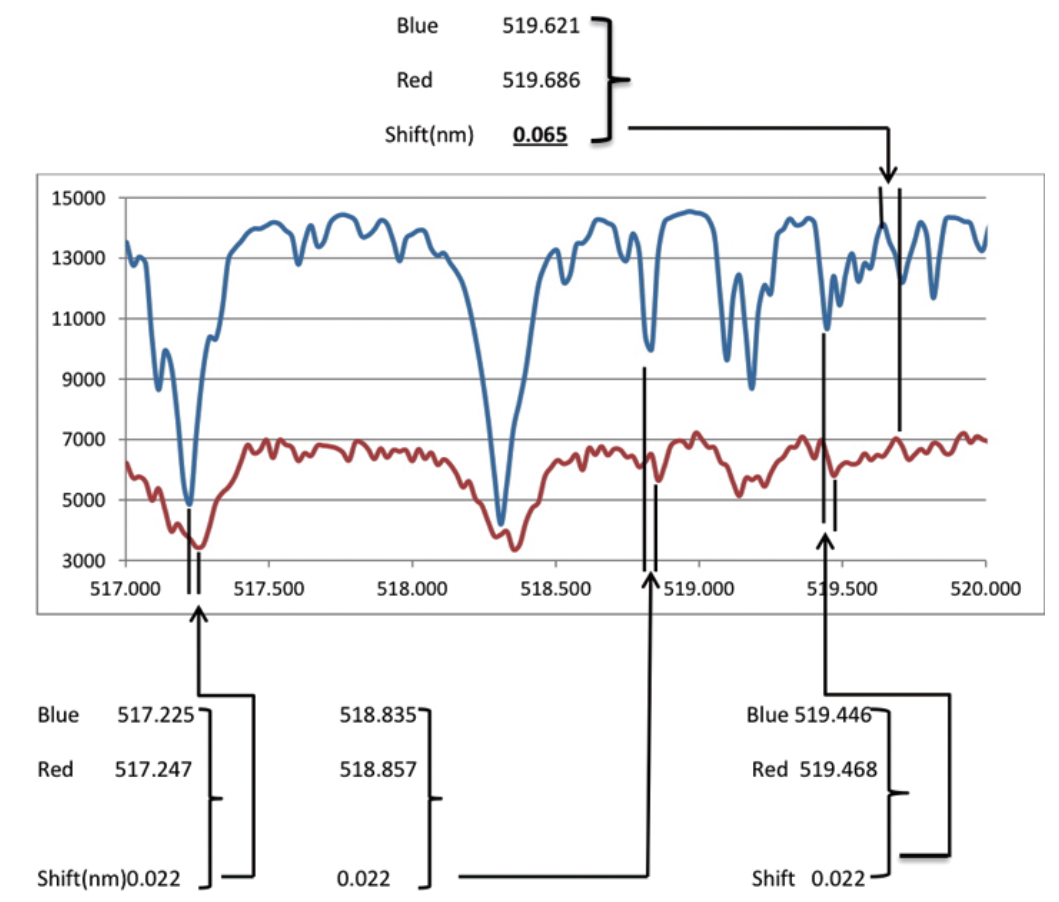

Fig. 8. Chromatographic analysis of a few wavelengths of direct visible Sunlight at about 70。 degrees elevation over the horizon obtained via the Yokogawa analyzer AQ63-73 on March 16-17, 2012, thus without any visible redshift, yet the IRS is detectable at that elevation, to become more and more undetectable for individual wavelengths with the decrease of the elevation.

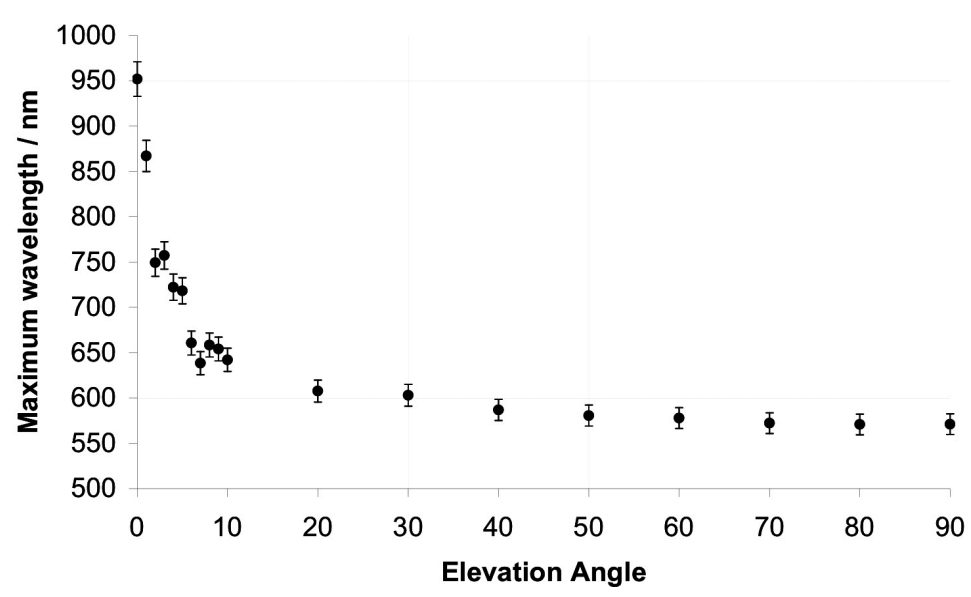

Fig. 9. Chromatographic analysis of all scans of direct Sunlight from the Zenith to the equator [19] obtained on March 16-17, 2012, via the Yokogawa analyzer AQ6373 confirming the existence of an IRS of at least $200 \mathrm{~nm}$ for the visible spectrum, plus additional IRS beyond that. the analysis has been obtained by fitting the data to a black body radiation curve (because that matches the spectrum shape better than a Gaussian shape) with black body temperature for the Sun of about $6000 \mathrm{~K}$. A large IRS corresponds to a proportional decrease in the temperature of the black body.

2. The increase of the IRS with air at increasing pressure established for the pipe [8-10] constitutes experimental evidence for the increase of the IRS of the Sun at Sunset with decreasing elevation over the horizon. In fact, the former establishes the increase of the IRS with the increase of 


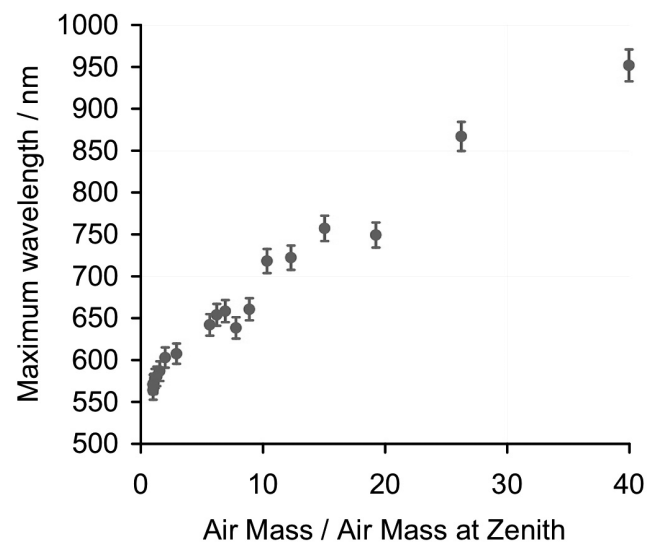

Fig. 10. Chromatographic analysis of all scans obtained via the Yokogawa analyzer AQ6373 of direct visible Sunlight from the Zenith to the horizon [19]. The vertical axis represents the maximal wavelength of the spectrum obtained by fitting an effective black body spectrum to the data. The horizontal axis represents the effective air mass (i.e. the path length of Sunlight) at a given elevation with respect to the path length when the Sun is at the Zenith. As one can see, this analysis confirms the increase of the IRS with the decrease of the elevation.

the trajectory of light in air, which increase is necessary for a quantitative representation of the evidence. Alternatively, any dismissal of the IRS origin of the increased redness of the Sun with the decrease of the elevation, to achieve credibility, must prove its compatibility with the increase of the IRS with the increase of the pressure in the pipe, which is another impossible task.

3. The establishing of the IRS in the pipe constitutes experimental evidence on the mutation of spacetime, under which the novel MS isogeometry, the LPS isosymmetry and isorelativity uniquely and unambiguously imply the IRS of the Sun at Sunset. Alternatively, by recalling that physical laws cannot be altered at will to meet pre-determined aims, any dismissal of the IRS of the Sun at Sunset, to achieve credibility, musty prove the absence of mutation of spacetime now established on numerous counts, which is another impossible task.

4. The very red color of the Sun at Sunset is evidence of its IRS origin. As shown earlier, red light cannot possibly reach us in the very long trajectory of the Sun at the horizon, thus leaving as the sole consistent origin the IRS of blue light all the way to red. Alternatively, any dismissal of the IRS for the Sun at Sunset, to achieve credibility, must prove that red light does indeed reache us when the Sun is at the horizon, which is yet another impossible task.

5. The large differences in cosmological redshifts for quasars and their associates galaxies [20] provide additional, this time astrophysical evidence of the IRS origin of the redness of the Sun at Sunset. In fact, the above outlined experimental evidence dismissing absorption and scattering as the origin of said largely different cosmological redshifts, leaves the IRS within different physical media as the sole quantitative representation [2b,10]. The IRS of the Sun at Sunset is then the unavoidable consequence. Alternatively, any dismissal of the IRS origin of the redness of the Sun at Sunset must prove compatibility with the absence of the IRS origin of said large differences in cosmological redshifts, which is yet another impossible task.

To confirm the IRS origin of the redness of the Sun at Sunset, on April 22, 2012, we conducted comprehensive confirmatory measurements via the Avantes wavelength analyzer model AvaSpec-ULS2048 from 380 to $1100 \mathrm{~nm}$. The measurements were conducted in the same location (St. Petersburg Beach, Florida West Coast), also from the Zenith to the horizon, and at the same sequence of elevation over the 


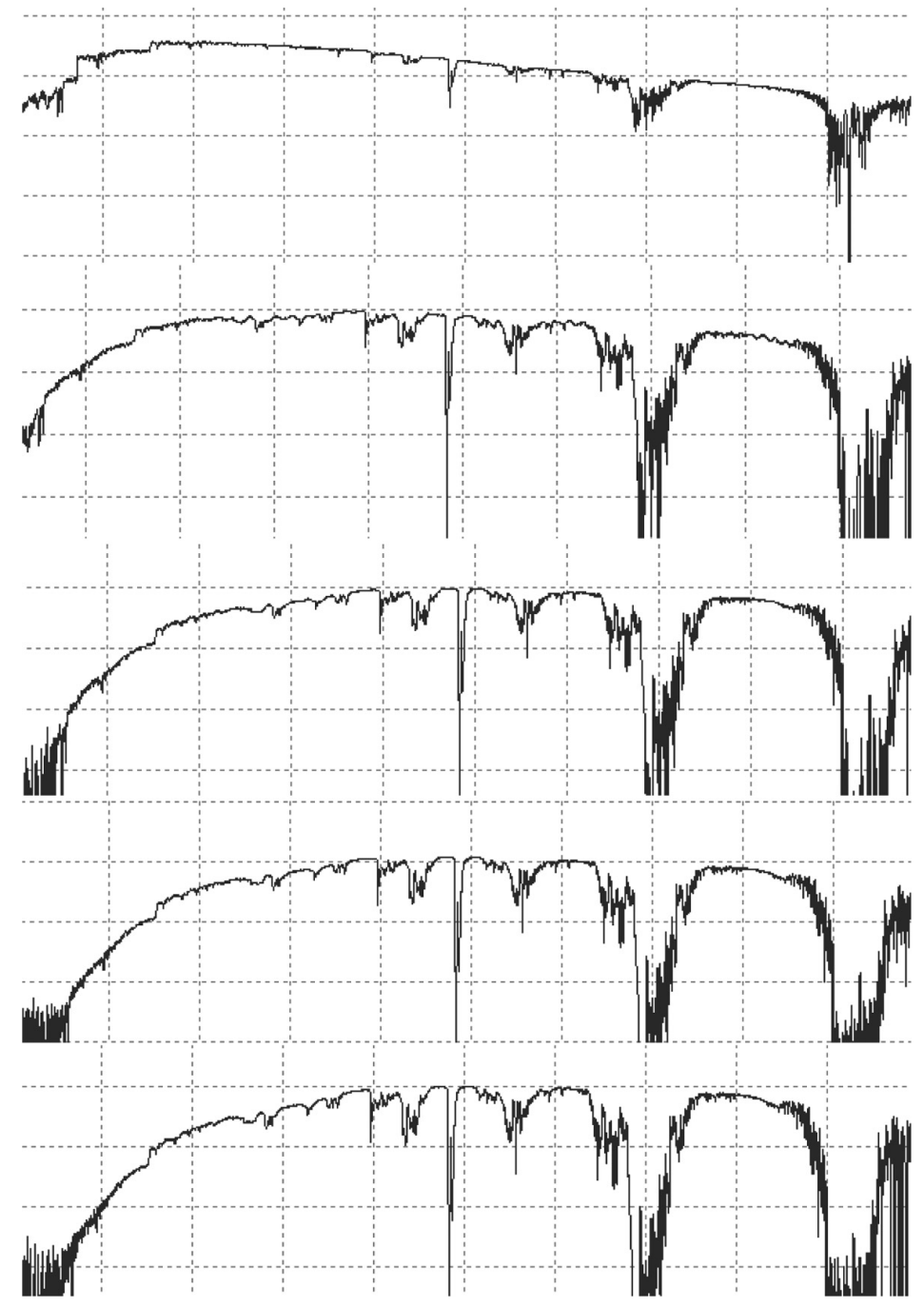

Fig. 11. Some of the chromatograms of the entire spectrum of direct Sunlight obtained with the Yokogawa analyzer AQ6373 with range of 350 to $1100 \mathrm{~nm}$ showing from the top down the spectrogram at the Zenith, $8^{\circ}, 3^{\circ}, 1^{\circ}$, and $0^{\circ}$ degrees elevations from the horizon used in the preceding analyses to confirm the primary origin of the IRS for the redness of the Sun at Sunset (comprehensive spectrograms, pictures and other information are available in Ref. [19]).

horizon as done for the Yokogawa analyzer. Analysis of the new raw data was also done with the same methods as those used for the preceding measurements.

The results of the new measurements are reported in Figs 12, 13, and 14, with all raw data and other information available for free pdf download from the link of Ref. [19]. As one can see, the Avantes analyzer shows quite clearly the shift of the "entire" spectrum of the Sun toward the red, exactly as it happens for the cosmological redshift of galactic light, thus providing a clear confirmation of its IRS origin. The new measurements also confirm the need for a quantitative and time invariant representation of a redshift of at least $200 \mathrm{~nm}$ as solely done to date in Section 2.6 below.

Finally, the new data clearly confirm the lack of frequency shift of the absorption lines as expected, because they are set by the chemistry of the atmosphere, thus confirming the dismissal of absorption as 

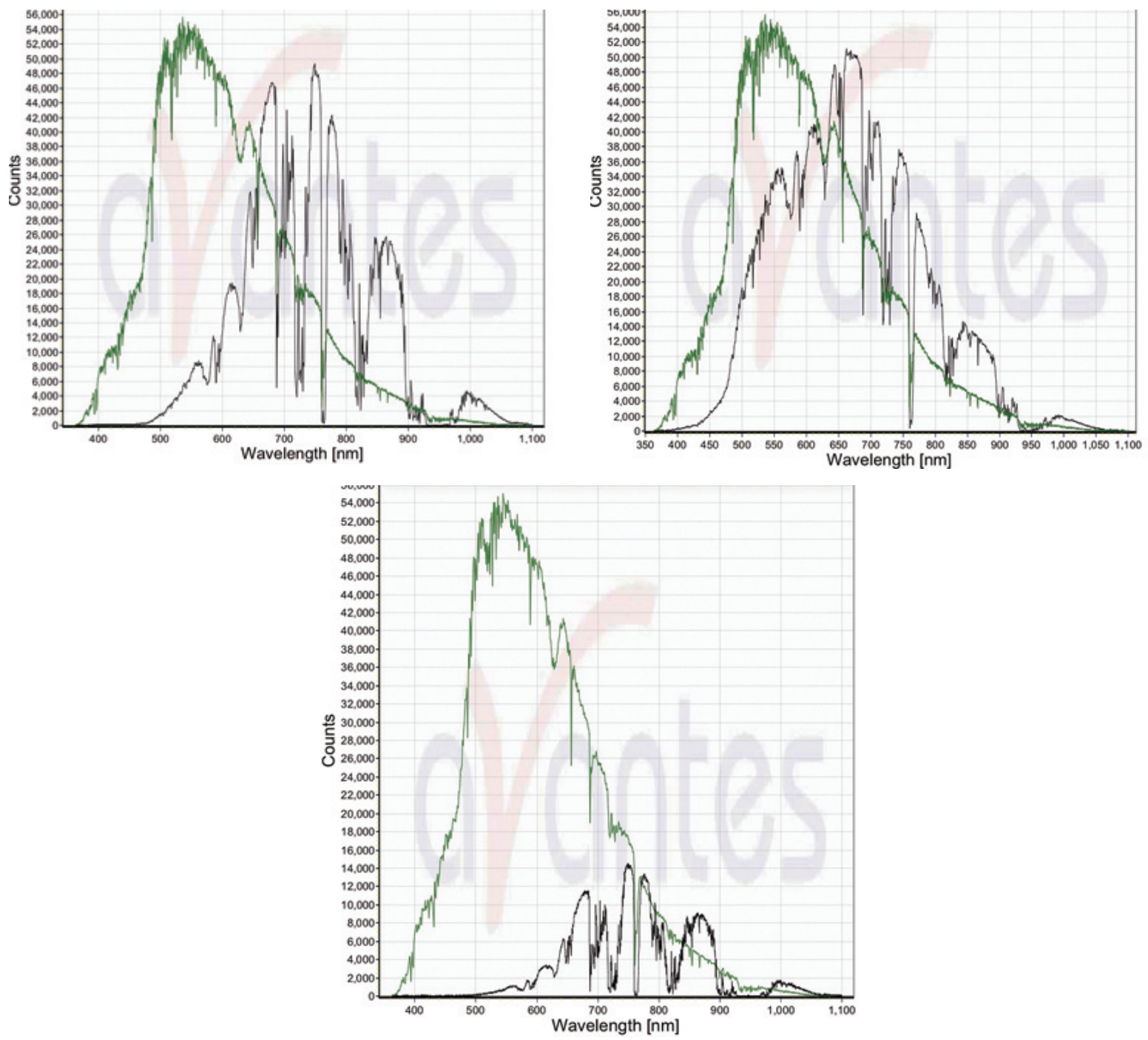

Fig. 12. Representative chromatographs of Sunlight from the Zenith to the horizon obtained on April 22, 2012, via the use of the Avantes wavelength analyzer model AvaSpec-ULS2048 clearly showing the shift toward the red of the entire spectrum of Sunlight, including the expected lack of frequency shift of absorption lines, thus confirming the preceding measurements via the Yokogawa wavelength analyzer model AQ6373.

the origin of the redshift of Sunlight at Sunset and Sunrise. The dismissal of scattering as the origin of the redshift of the Sun requires no comments since established at both the astrophysical [11] and laboratory [10] levels, besides the notorious impossibility for scattering to allow a scientific, that is, quantitative representation of frequency shift of any appreciable value, let alone for at least $200 \mathrm{~nm}$.

\subsection{Conventional Doppler contributions}

It should be mentioned that the conventional Doppler law does indeed contribute to the redness of the Sun at the horizon for the evident reason that, even though the distance between Earth and Sun can be assumed as remaining constant during the few hours of the measurements, Earth rotates and, therefore, 


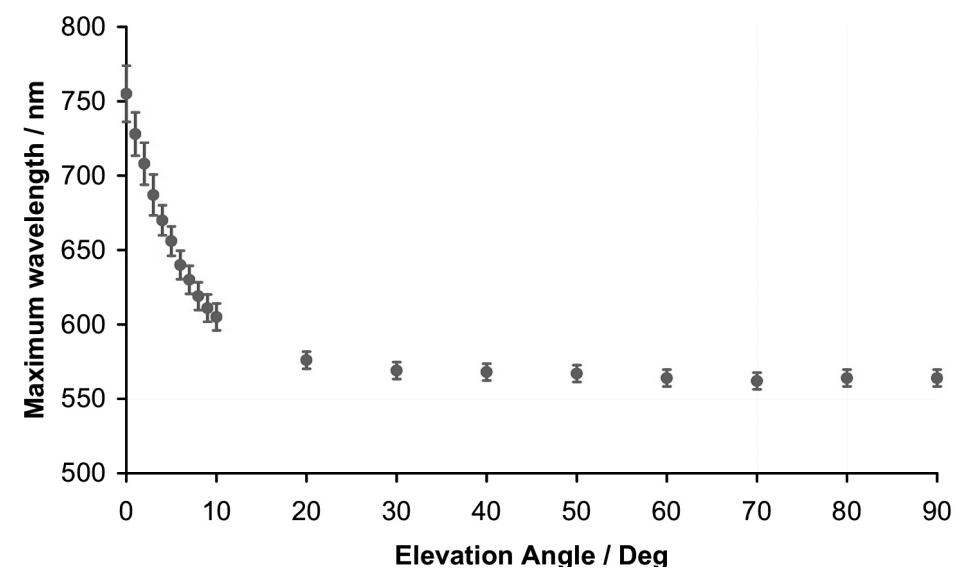

Fig. 13. Elaboration of the data of Fig. 12 via the Avantes wavelength analyzer AvaSpec-ULS2048 confirming the elaboration of Fig. 9 via the Yokogawa analyzer AQ6473. Note the confirmation of the need for a quantitative and time invariant representation of a redshift of at least $200 \mathrm{~nm}$ as solely reached to date in Section 2.6.

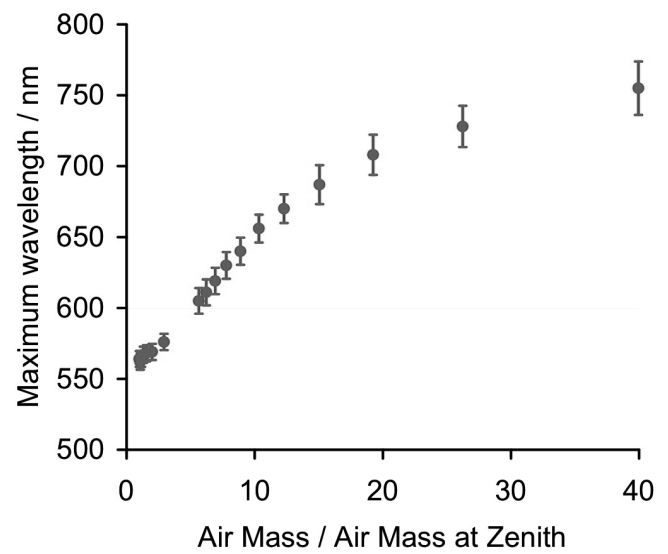

Fig. 14. Elaboration of the data of Fig. 12 via the Avantes wavelength analyzer AvaSpec-ULS2048 confirming the elaboration of Fig. 10 via the Yokogawa analyzer AQ6473. Note the confirmation again of the need for a quantitative and time invariant representation of a redshift of at least $200 \mathrm{~nm}$ as solely reached to date in Section 2.6.

an observer on the surface of Earth moves away from the Sun at Sunset and toward the Sun at Sunrise, thus causing a conventional Doppler shift.

However, simple calculations from known data (radius of Earth and rotation time) show that the Doppler contribution is quite small. In fact, the approximate tangential speed of Earth at the equator has the value

$$
v_{\text {tang }} \approx 430 \mathrm{~km} / \mathrm{s} \text {. }
$$

As a consequence, we have the following approximate value (also at the equator) of the Doppler shift term

$$
\frac{v}{c}=1.43 \times 10^{-6},
$$

thus being definitely insufficient to provide a numerical representation of the redness of the Sun at Sunset and Sunrise. 
It is intriguing to note that, despite its small value, the conventional Doppler shift is indeed visible to the naked eye. By recalling that such a contribution is a redshift at Sunset and a blueshift at Sunrise, the comparison of the redness of the Sun at Sunset and Sunrise shows indeed a difference visible to the naked eye which is precisely due to the opposite Doppler contributions.

\subsection{Quantitative and time invariant IRS representation}

We now outline the representation of the IRS of the Sun verifying the conditions identified at the beginning of this sections, namely: 1) Being capable of representing the increase of the IRS with the increase of the distance $d$ traveled by light in our atmosphere; 2) Being capable of representing as a limit the IRS of the light wavelength from the blue, $\lambda_{b l u e} \approx 450 \mathrm{~nm}$, to the red, $\lambda_{\text {red }} \approx 650 \mathrm{~nm}$; and 3) All representations being invariant over time in the indicated sense. Note that the selected value of $200 \mathrm{~nm}$ for the IRS is an upper limit selected primarily to illustrate the representational capabilities of IsoRelativity.

By recalling that the Doppler effect contributes to the redness of the Sun at the horizon, we have to use the Doppler-Santilli isolaw (5) which we write for the wavelengths

$$
\lambda_{\text {irs }}=\lambda_{\text {blue }} /\left(1-\frac{v}{c} \frac{n_{4}}{n_{s}}\right)
$$

where: $\lambda_{i r s}$ is the increase of $\lambda_{b l u e}$ due to the IRS for a given distance $d ; n_{s}$ is the space characteristic quantity of the atmosphere in the selected direction $s ; n_{4}$ is the local index of refraction of light in air; the local speed of electromagnetic (e.g., radio) waves is the familiar expression $C=c / n_{4} ; v$ is the tangential speed of the observer due to the rotation of Earth; $c$ is the speed of light in vacuum; and we have only selected the minus sign because of the dominance of the IRS, the Doppler blue shift at Sunrise being considered below.

Since the IRS increases with the increase of the distance covered by light in air, we can correctly assume the following expansion in terms of $+d$ for the Sunset

$$
\frac{n_{4}}{n_{s}} \approx 1+K_{s s} d+\ldots
$$

where: $K_{s s}$ can be well approximated into a constant for Sunset (ss) with dimension $\mathrm{km}^{-1}$ when $d$ is measured in $k m$, and Eq. (6) becomes

$$
\Delta \lambda=\lambda_{i r s}-\lambda_{b l u e}=\frac{v}{c}\left(1+K_{s s} d+\ldots\right) \lambda_{i r s}=K_{s s} d \frac{v}{c} \lambda_{i r s}
$$

because, as indicated earlier, the conventional Doppler's effect is dramatically smaller than the IRS, thus being ignorable with respect to the latter. Evidently, for $d=0$ but $v \neq 0$ we have

$$
\Delta \lambda_{d=0}=0,
$$

as desired because we are dealing with the IRS and not the conventional Doppler shift.

To get a representative numerical value of $K$, we assume as an average expression at the horizon and at the equator from Eqs (6) and (8)

$$
\Delta \lambda=K_{s s} d \frac{v}{c} \lambda_{i r s}=K_{s s} 70001.4310^{-6} 650 \mathrm{~nm}=200 \mathrm{~nm} .
$$


from which we reach the desired numerical value

$$
K_{s s}=30.7 \mathrm{~km}^{-1}
$$

It should be noted that in expansion (7) we could have assumed in first approximation $n_{4} \approx 1$ and $C \approx c$ in air, although we have preferred to maintain $n_{4}$ as part of the expansion because the index of refraction in air varies indeed with the variation of $d$.

For the case of the Sunrise, instead of Eq. (6), we have the expression

$$
\lambda_{\text {irs }}=\lambda_{\text {blue }} /\left(1+\frac{v}{c} \frac{n_{4}}{n_{s}}\right)
$$

since the observer moves toward the Sun, thus causing a conventional Doppler blueshift. But the distance traveled by light in air decreases over time and, consequently, we can assume the expansion of $n_{s} / n_{4}$ in terms of $-d$. In this case, we reach the following expressions in lieu of Eq. (6)

$$
\lambda_{\text {irs }}=\lambda_{\text {blue }} /\left(1+\frac{v}{c} \frac{n_{4}}{n_{s}}=\left(1+\frac{v}{c}\left(1-K_{s r} d+\ldots\right) \lambda_{\text {irs }} \approx\left(1-K_{s r} d \frac{v}{c}\right) \lambda_{i r s} .\right.\right.
$$

where $K_{s r}$ is now the new constant for Sunrise (sr), from which we obtain the expression equivalent to that for the Sunrise

$$
\Delta \lambda=\lambda_{\text {irs }}-\lambda_{\text {blue }}=-\frac{v}{c}\left(1-K_{s r} d+\ldots\right) \lambda_{\text {irs }}=K_{s r} d \frac{v}{c} \lambda_{\text {irs }}=200 \mathrm{~nm} .
$$

thus providing a numerical value of the constant $K_{s r}$ for Sunrise very close in values to that for $K_{s s}$ (although slightly smaller due to the opposite Doppler contributions here ignored.

It is evident that a numerical representation for the IRS in the pipe of Refs [8-10] without Doppler's effect is considerably simpler than the above and it is therefore ignored.

We complete in this way the desired illustration that the IRS formalism can provide a numerical representation of the increase of the redness of the Sun with the increase of the travel of light in air for both Sunset and Sunrise, as well as the capability of representing large IRS values of the order of $200 \mathrm{~nm}$ because needed to represent visual evidence, with the understanding indicated above that the achievement of its exact numerical value will require decades of additional experimentations left to interested colleagues.

The invariance over time of the numerical predictions is assumed by the universal Lorentz-PoincaréSantilli isosymmetry of basic invariant (1) from which the IRS law (6) follows uniquely and unambiguously.

It should also be noted that supporters of the absorption and scattering conjectures as the origin of the redness of the Sun at the horizon, to achieve some credibility, are requested to prove that said conjectures can indeed achieve the same representation as that achieved by the IRS formalism, including the representation of redshifts of the order of $200 \mathrm{~nm}$ as required by visual evidence, which is notoriously impossible.

Note that the measurements presented in this section may likely require a reinterpretation of a number of planetary data, such as the redness of Jupiter's atmosphere may well result as being due to the IRS, rather than (or in addition to) its chemical composition.

al comment, recall that the frequency shift of the Sun at Sunset on Earth cannot be toward the blue because light travels through air mostly at low temperature. Nevertheless, Santilli [5] predicts that Starlight can indeed be shifted toward the blue at the horizon in planets whose atmosphere is sufficiently $h o t$, as expected for planets sufficiently close to a star. 


\subsection{Preliminary implications}

It is evident that the measurements of deviations from special relativity within our inhomogeneous and anisotropic atmosphere provide experimental support for the deviations from special relativity in rotating discs measured by A. Kholmetskii et al. [21].

Recall also that Einstein general relativity represents the gravitational field of Earth as being homogeneous and isotropic. The IRS measurements presented in this paper establish that such a conception of gravitation can at best be approximate due to the evident anisotropy of Earth, its lack of perfectly spherical character, and other reasons. Consequently, the IRS measurements presented in this paper provide experimental support to the measurements of the anisotropy of the gravitational field of Earth predicted by Santilli [5,6] and first experimentally verified by R/Verrault [22].

Said anisotropy is also expected to imply different IRS for different latitudes. For instance, the Sun IRS measured at a given latitude in the Northern hemisphere of Earth may well be different than the Sun IRS measured at the conjugate latitude in the Southern hemisphere, although the confirmation of denial of such a difference may well require decades of progressively more accurate measurements due the small value of the possible difference.

Note that the index of refraction $n_{4}$ in isospacetime (1) remains completely unrestricted by isoformulations, thus being either smaller or bigger than 1 depending on the medium considered. In air, we evidently have $n_{4}>1$ and, therefore, $C=c / n_{4}<c$ due to the low density of our atmosphere. However, the values $n_{4}<1, C=c / n_{4}>c$ are predicted for suitable interior dynamical conditions without any violation of causality or other physical laws. This is due, on one side, to the inapplicability of special relativity within physical media and, on the other side, the validity of the covering isorelativity and its Lorentz-Poincaré-Santilli isosymmetry for which speeds within physical media are arbitrary.

As an illustration, recall that a proton in a particle accelerator requires infinite energy to reach the speed of light, since the only applicable accelerating forces are of potential type. The situation for the same proton in the core of a star, or within a physical medium at large, is dramatically different because in the latter case accelerations are dominated by contact, zero range forces for which conventional energy considerations have no physical meaning. Consequently, the inapplicability of special relativity within our atmosphere established in this paper provides experimental support to:

1) The superluminal communications first measures by G. Nimtz in 1992 (see the latest paper [23] and references quoted therein), in which superluminal speeds are achieved by propagating electromagnetic waves within certain special guides, thus characterizing interior dynamical conditions with general isospacetime (1). In particular, isorelativity allows Nimtz superluminal communications to verify fully causality and other physical laws.

2) The superluminal speeds announced by the CERN/GRAN SASSO laboratories in September 2011 [24] for neutrinos traveling underground, thus characterizing again strictly interior dynamical conditions for which isorelativity applies fully, while special relativity is inapplicable for an endless number of reasons, e.g., the impossibility underground of propagating light. It is unfortunate for mankind that organized interests on Einstein have prevented due scientific process on paper [24] until now (Spring 2012), which is solely achievable via its publication and then the publication of dissident view in refereed journals. By contrast, organized interests on Einstein have preferred releasing hypothetical objections in blogs and other non-scientific conduits.

3) The expulsion of large masses by galactic centers that have been measured for decades as occurring at speeds bigger than the speed of light in vacuum (see, e.g., Ref. [25]). It is unfortunate for mankind that due scientific process in these important measurements has been also suppressed 

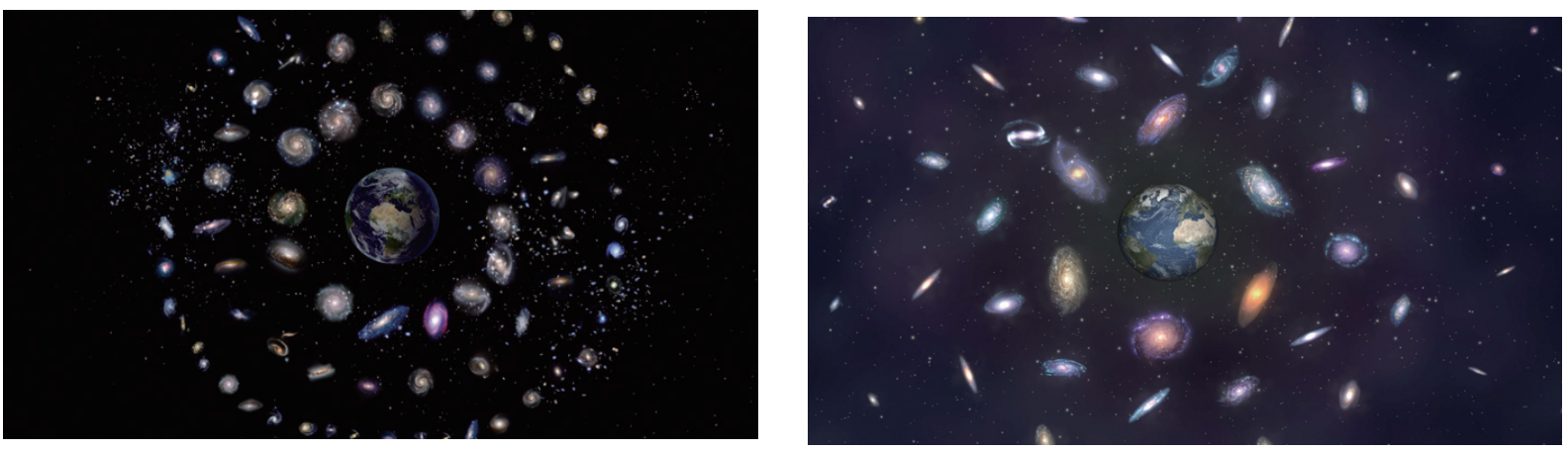

Fig. 15. An artist's rendering of the Middle Age conception of Earth at the center of the universe (left view), and an illustration of the contemporary conception of the expansion of the universe (right view) with the generally unspoken implication of Earth again at the center of the universe, since Hubble's law implies the same cosmological redshift for all galaxies with the same distance from Earth in all directions in space and such a redshift increases with the increase of the distance from Earth.

by organized interests on Einsteinian theories, again, via the use of special relativity arguments, when the expulsion occurs under huge contact, zero-range forces for which special relativity has no known meaning of any type.

The most important implication of the IRS origin of the redness of the Sun at the horizon is that of providing an additional experimental evidence, besides the considerable evidence provided in Vol. IV of Refs [4], of the mutation of spacetime caused by the presence of energy at large or matter in particular, in favor of isorelativity and its underlying isoformulations.

A most visible implication is that the existence of measurable deviations from the Minkowski spacetime within a physical medium so weakly dense as our atmosphere evidently implies the existence of much bigger deviations within the hyperdense medium in the scattering region of high energy particle experiments. The additional admission that such events are highly irreversible over time, compared to the strict reversibility of special relativity, requires a number of rather serious revisions of 20th century particle physics, such as the lack of existence of the Higgs boson, the impossibility for quarks being physical particles in our spacetine, and other revisions [4].

In any case, the inevitable mutation of the Minkowskian spacetime in the hyperdense regions of high energy scattering processes directly implies the lack of exact character of 20th century scattering theories in favor of the covering nonunitary Lie-isotopic and Lie-admissible scattering theories of hadronic mechanics [26], with consequential need for a re-inspection of most results claimed under the unspoken belief of the exact validity of Einsteinian theories for inelastic, thus irreversible high energy scattering processes.

All in all, it is not inconceivable that the measurements of IRS and IBS presented in this and in the preceding papers [8-10] may one day signal the birth of a new technology allowing the control of the characteristics of light in essentially the same way we can nowadays control the characteristics of sound. In this case, we would have a number of scientific, industrial and medical applications, such as the conceivable achievement of under cutaneous operations or destruction of tumors without skin cuts merely achieved via the optimization of resonating effects for intersecting laser beams below the human skin. 


\section{Experimental confirmation of the absence of universe expansion and related cosmological conjectures}

In 1929, Edwin Hubble [27] discovered the cosmological redshift according to which light reaching us from distant galaxies is redshifted in an amount which is the same for all galaxies having the same distances from earth in all space directions, and said redshift increases with the increase of the distance from us.

Immediately following Hubble's discovery, Fritz Zwicky [28] and others submitted the hypothesis of the "tired light", according to which the cosmological redshift is due to loss of energy E - hv by light when scattering through intergalactic gases during the long travel to reach Earth.

However, it was already known in the astrophysics community of the early 20th century (but not so by the atmospheric physicists of the late 20th century) that scattering of light in a gas cannot cause frequency shift, as experimentally confirmed in our laboratory on Earthm Ref. Ref. [10], and at the astrophysical level, Ref. [11]. Consequently, despite important supporters (such as Hubble himself, de Broglie, Fermi and others), the scattering origin of the cosmological redshift was dismissed.

The above occurrences left no other choice than the assumption of the exact validity of special relativity throughout the universe, with consequential chain of

\subsection{Contemporary cosmological conjectures}

Conjecture 1: The universe is expanding according to Doppler's shift law [29,30], thus implying that Earth is at the center of the universe since, from Hubble's law, the speed of galaxies moving away from Earth is the same for the same distances in all directions in space.

Conjecture 2: The expansion of the universe increases with the increase of the distance from Earth 9 see [31], thus implying again that Earth is at the center of the universe since, also from Hubble's law, the acceleration of the expansion is the same for the same distances in all directions in space.

Conjecture 3: The universe was born in a primordial explosion, the "big bang", occurred some 13,7 billions years ago (see, e.g., Ref. [32]). This conjecture was voiced for the intent of maintaining the credibility of the expansion of the universe, thus implying that the claimed primordial explosion had to occur in the galactic vicinity of Earth and, consequently implying again that Earth is at the center of the universe.

Conjecture 4: Space itself is expanding thus carrying along all galaxies (see, e.g., Ref. [33]). This additional conjecture was voiced to prevent the generally unspoken return to the Middle Ages with Earth at the center of the Universe. However, the conjecture still implies Earth at the center of the universe because it cannot possibly include the acceleration of the expansion that remains the same for the same distances in all directions ion space.

Conjecture 5: The dynamics of galactic stars depends on a mysterious invisible substance, the "dark matter", surrounding a galaxy [34]. This additional conjecture originated from the claimed equal velocity of stars with the increase of the distance from the galactic center.

Conjecture 6: The dynamics of the entire universe depends on yet an additional invisible substance, the "dark energy", constituting over 95\% of the energy in the universe (see e.g., Ref. [36]). This yet additional conjecture was voiced for the generally unspoken insufficiencies of the big bang conjecture (some of which are recalled below), to explain the expansion and the acceleration of the expansion of the universe, with ensuing need for enormous amounts of energy.

Conjecture 7: The speed of light in vacuum is the maximal causal speed throughout the universe, thus including within intergalactic spaces as well as in the interior of stars, quasars and black holes. This 
yet additional conjecture is generally unspoken, but it is at the foundation of most astrophysical and cosmological calculations conducted in the preceding conjectures e.g., to predict the energy equivalence of a black hole.

The comprehensive measurements presented in this paper have established, apparently for the first time, that Zwicky's main idea on the origin of the cosmological redshift, loss of energy by light during its travel throughout intergalactic gases [28], is indeed correct, although realized with a mechanism unknown during Zwicky's time, and today known as Santilli9i IsoRedShift. The experimental confirmation of Zwicky's original hypothesis then implies a consequential predictable revision of the above cosmological conjectures.

To understand the content of this section, the reader should always keep in mind that, whether intentional or de facto, the main implication of all the above conjectures is preserving the validity of Einsteinian theories throughout the universe. The reader should also keep in mind that, since none of the above conjectures is expected to receive clear experimental verifications, and they remain with basic insufficiencies, additional conjectures in support of preceding unverifiable conjectures are under way. For instance, the voicing of the conjecture of yet an additional mysterious particle called the "neutralino" is under way due to the inability to reach an experimental verification of the conjecture of dark matter, and so on.

As stated in Refs [8,9], under the conditions that physical laws have to be the same throughout the universe and cannot be modified to maintain Einsteinian theories, the redness of the Sun at Sunset illustrated in Fig. 1 and its IRS origin constitute visual and direct or indirect experimental evidence, respectively, of the absence of all the above Conjectures 1 to 7 . The IRS measurements of direct Sunlight at Sunset presented in this paper merely provide a confirmation of these new cosmological vistas.

To appraise the complexity of the issue, it should be indicated that the elimination of all these far reaching and still growing conjectures required over thirty years of mathematical, theoretical and experimental research [1-9] to establish the inapplicability of special relativity within physical media and construct covering theories. Hence, the elimination of all Conjectures 1 to 7, as well as the prevention of additional extreme conjectures, crucially depends on the inapplicability of Einsteinian theories at large cosmological distances for which physical media cannot be ignored, with the understanding that special relativity remains valid at local distances for point particles and electromagnetic waves moving in vacuum. The cosmological implications of these studies can be outlined as follows:

\subsection{Absence of universe expansion, acceleration of the expansion, big bang and space expansion}

As well known since the time of Hubble's discovery, intergalactic space is far from being empty, since it is constituted by a medium with high energy density characterized by light (originating from all of the universe), gases, particles, radiations, etc. As established experimentally on Earth [8-10], such a medium causes a mutation of spacetime into the Minkowski-Santilli isospacetime, with consequential inapplicability (at said intergalactic distances) of special relativity in favor of the covering isorelativity and resulting IRS without a necessary relative motion between the source, the medium and the observer (see below for the case with $v \neq 0$ ). It is evident that the IRS eliminates any need for the expansion of the universe since light merely loses energy $E=h \nu$ to the very cold intergalactic medium, resulting in a numerical and time invariant (thanks to the LPS isosymmetry), representation of the cosmological redshift of galaxies without any necessary motion away from us. But the IRS is proportional to the distance traveled by light in the medium, as illustrated in Fig. 1 and expansion (7), and this property eliminates the need for the acceleration of the expansion. The lack of any need for the biog bang is then 
so evident not to require comments. Finally, the elimination of the expansion of the universe avoids the return to the Middle Ages with Earth being at the center of the universe, with consequential lack of need for the very far reaching, thus highly implausible conjecture that space itself is expanding.

The incompatibility of the big bang conjecture with experimental evidence on the structure of the universe should be recalled. As it is the case for all explosions without adaptations to preferred theories, the universe around Earth should be completely empty of any galaxy for up to 13.7 billion light years, and the "debris" of the claimed explosion (the galaxies) should slow down with the increase of the distance due to inevitable intergalactic dust. Both incontrovertible implications of any explosion, including the big bang, are dramatically disproved by astronomical evidence.

Furthermore, the conjecture that the background radiation is "evidence" of the big bang has been disproved by calculations showing that, due to its weakness, such a radiation should have been absorbed by intergalactic media and galaxies billions of years ago. Consequently, the plausible representation of the background radiation requires a continuous source of energy for its very existence. The needed continuous source is provided precisely by the IRS because the energy transferred by light to intergalactic media cannot be lost, thus manifesting itself in the form of a faint radiation filling up the entire universe in an almost isotropic fashion, because the light originating from galaxies propagates and experiences the IRS throughout the universe.

We should also mention the implausibility of the expansion of the universe because the speed needed for the representation of the cosmological redshift of galaxies at the edge of the known universe is today approaching and then surpassing the speed of light in vacuum, namely, the conjecture here considered implies that billions of galaxies travel at superluminal speeds! Of course, such an occurrence is quickly bypassed with the additional far reaching conjecture that space itself is expanding so as to maintain the validity of Einsteinian theories in the universe. However, serious scholars are expected to admit that an extremely far reaching conjecture (billions of galaxies traveling at extreme speeds away from us) cannot be credibly cured with yet another extremely far reaching conjecture (space itself is expanding throughout the immense distances of the universe at speeds that may surpass that of light in vacuum).

For a numerical and time invariant IRS representation of the cosmological redshift we refer interested readers, for brevity, to Ref. [8].

\subsection{Absence of dark matter}

The sole experimental evidence we have on innergalactic dynamics is the redshift of star lights which is anomalous because essentially the same with the decrease of the distance from galactic centers (the decreased centrifugal speeds of stars while approaching the galactic center, and consequential decrease of their speed, was expected to imply a corresponding decrease of their redshift). Following the tacit assumption of the exact validity of special relativity within the innergalactic media and the additional tacit assumption of the exact validity of the Doppler shift in said media, Vera Rubin [34] assumed that peripheral stars in a galaxy have "anomalous velocities". The attempts at explaining such a conjecture then stimulated the further far reaching Conjecture 5 on the mysterious and invisible dark energy.

However, innergalactic spaces are also far from being empty, since they are filled up with gases, dust, particle, etc. according to evidence visible in telescopes. Additionally, the density of innergalactic media increases with the decrease of the distance from the galactic center. The IRS interpretation of the anomalous redshift of peripheral galactic stars is then immediate without any need of conjecturing a hypothetical and invisible substance. In fact, the measurements of Refs [8-10] as well as of this paper establish that the IRS is proportional to the density of the medium. Therefore, in peripheral galactic stars 
the redshift remains essentially the same with the decrease of the distance from the galactic center due to the corresponding increase of the density and, therefore, of the IRS. Note that in this case the stars have motion. Therefore, the applicable law is the Doppler-Santilli IsoLaw (2) with a generally non-null speed. For a numerical and time invariant representation of galactic dynamics without the conjecture of the dark matter, we refer, for brevity, to Ref. [8]. It should be indicated that these results provide direct experimental verifications via measurements on Earth of M. E. de Souza model of galactic dynamics without dark matter [35].

Independently from the above evidence, the implausibility of the dark matter conjecture should be noted. In the event dark matter is uniformly distributed within a galaxy, no effect in the dynamics of galactic stars is conceivably possible. The only possibility for the mysterious dark matter to have any effect for a given star is that it is non-uniformly distributed as needed, e.g., in front of a star, but then the behavior of a nearby star becomes non-physical, Consequently, the far reaching conjecture of the dark matter has indeed achieved the unspoken objective of derailing attention from the expected inapplicability of Einsteinian theories in the interior of galaxies, but said conjecture has failed to achieve a consistent representation of the anomalous redshift for which it was intended.

\subsection{Absence of dark energy}

Following the series of preceding far reaching conjectures, each without serious hopes of clear experimental verifications, each conjecture being voiced to support a preceding unverifiable conjecture, the voicing of the yet additional conjecture of the dark energy has caused concern in the silent majority of the scientific community because excessively far reaching, inasmuch it implies that all the billions of visible galaxies essentially have no major impact in the dynamics of the universe, since $95 \%$ of the needed energy is believed as being provided by a mysterious and invisible energy pervading the entire universe.

Said concern has a number of motivations, such as: the conjecture of dark energy has been voiced in support of the preceding far reaching conjecture of the acceleration of the expansion of the universe, since the latter required an amount of energy simply beyond our imagination; the relationship between the conjecture of dark energy and the conjecture of the big bang has remained essentially unspoken, since the claim of the representation of the expansion of the universe via the big bang has remained untouched following the conjecture of dark energy; as it was the case for the dark matter, a uniformly distributed dark energy cannot provide any measurable effect on the dynamics of an individual galaxy; it is well known by experts that energy (and not the mass) is the very source of gravitational attraction according to Einstein general relativity, as a result of which the explanation of the acceleration of the expansion of the universe (rather than the expected contraction due to gravitational attraction) requires yet additional theoretical conjectures for the dark energy to achieve compatibility with Einstein gravitation; and other reasons.

It is evident that the elimination of the expansion of the universe and of the acceleration of the expansion eliminate any need for the far reaching conjecture that $95 \%$ of the energy of the universe is contained in the mysterious and invisible dark energy. The reader should be aware that the very physical laws used for the calculations on dark energy are conjectural themselves, since they are based on the additional conjecture that the speed of light in vacuum is the maximal causal speed inside stars, quasars and black holes (the latter conjecture is evidently necessary to calculate the energy equivalence of the masses).

Albert Einstein formulated his celebrated mass-energy equivalence principle $E=m c^{2}$, specifically, for point particles moving in vacuum because, under these conditions, $c$ is indeed the maximal causal 
speed for the mass considered. By contrast, calculations leading to the conjecture of dark energy are based on extending the applicability of the same equivalence law to large hyperdense astrophysical bodies, such as stars, quasars and black holes, for which it is known today that $c$ cannot be the maximal causal speed (e.g., for the interior of black holes when all conventional laws are known to fail). In fact, the mutation of spacetime caused by hyperdense matter evidently mutates the maximal causal speed into a covering notion. Consequently, the very basic law used in the calculations on dark energy are highly questionable and are actually disproved by available fits of experimental data in particle physics experiments. For brevity, we refer the interested reader to paper [8] for calculations based on the IsoEquivalence Principle, and Refs [2-4] for detailed treatments.

\subsection{Concluding remarks}

It is hoped that the most orthodox physicists will see the need for a structural revision of 20th century cosmology since for their own credibility and interests the current ever growing chain of unverifiable conjectures definitely will not resist the test of time. We should mention in this respect that Santilli IsoCosmology for matter (see Vol. IV of Refs. [4]) avoids all Conjectures 1 to 7 via the use of physical laws first verified in our laboratory on Earth according to Galileo's teaching. The laws are then applied to the universe. Incidentally, said cosmology essentially implies a return to the the historical Steady State Cosmology although with 21 st century advances, such as continuous creation via the transfer of energy from the ether to our physical world in the synthesis of the neutron from the hydrogen atoms in the core of stars (see Ref. [37] and preceding literature quoted thereafter).

In closing, we would like to present a few comments illustrating the complexity of the universe. Recall that Einstein special and general relativities cannot represent the conjugation from neutral matter to neutral antimatter as needed for the study of antimatter in the universe. This structural insufficiency has stimulated the construction of Santilli isodual theory of antimatter [38] that does indeed provide the missing conjugation for neutral matter to antimatter, but a necessary consequence is the gravitational repulsion between matter and antimatter galaxies.

Another necessary consequence is a physical distinction between light emitted by matter and that emitted by antimatter, known as the isodual light. For instance, the isodual light is predicted to be repelled by a matter gravitational field and have anomalous refractions on matter lenses (the angle of refraction of isodual light in transparent matter is the conjugate of that for matter light in a matter medium). Consequently, there are serious doubts on the efficiency of our telescopes to detect antimatter stars and galaxies [39,40]. It is then realistically possible that antimatter galaxies could indeed exist in our universe because they are not necessarily visible to us via our current observational means. In any case, our planet has been devastated in the past by antimatter asteroids annihilating in our atmosphere without leaving any crater in the grounds, such as the known 1927 Tunguska explosion in Siberia.

In conclusion, at the current state of our rather limited knowledge of the universe, we cannot exclude the existence of antimatter galaxies interspersed with matter galaxies, in which case the gravitational repulsion between matter and antimatter galaxies might well imply the expansion of the universe, although at such speeds to cause cosmological redshifts dramatically smaller than those currently measured due to the vast distances between galaxies and the consequently very weak character of the gravitational repulsion.

\section{Acknowledgments}

The authors express special thanks to J. V. Kadeisvili for an accurate control of the manuscript and numerous objections, R. Verreault for very advanced and penetrating comments on the scattering profile 
of the reported measurements, and I. Gandzha because his correct criticism on the inconclusive IRS origin of the redness of Sun at Sunset, when restricted to reduced spectrum of Fig. 4, was important to motivate additional measurements reported in Figs 6-14. Thanks are also due to V. de Haan, H. Ahmar, C. Corda, A. Kholmetskii and other colleagues for invaluable critical comments and suggestions. Final thanks are due to Mrs. Dorte Zuckerman for the linguistic review of the manuscript, although the authors are solely responsible for its content due to numerous editorial modifications and clarifications enacted in the final version.

\section{References}

[1] R.M. Santilli, Lie-isotopic Lifting of Special Relativity for Extended Deformable Particles, Lettere Nuovo Cimento 37 (1983), 545. http://www.santilli-foundation.org/docs/Santilli-50.pdf.

[2] R.M. Santilli, Isotopic Generalizations of Galilei and Einstein Relativities, Vols. I [3a] and II [3b] (1991), International Academic Press http://www.santilli-foundation.org/docs/Santilli-01.pdf. http://www.santilli-foundation.org/docs/ Santilli-61.pdf.

[3] R.M. Santilli, Elements of Hadronic Mechanics, Volumes I [4a] and II [4b], Ukraine Academy of Sciences, Kiev, second edition 1995. http://www.santilli-foundation.org/docs/Santilli-300.pdf; http://www.santilli-foundation.org/docs/Santilli301.pdf.

[4] R.M. Santilli, Hadronic Mathematics, Mechanics and Chemistry, Volumes I, II, III, IV and V International Academic Press 2008, http://www.i-b-r.org/Hadronic-Mechanics.htm.

[5] R.M. Santilli, Isominkowskian Geometry for the Gravitaitonal Treatment of Matter and its Isodual for Antimatter, Intern J Modern Phys D 7 (1998), 351. http://www.santilli-foundation.org/docs/Santilli-35.pdf.

[6] R.M. Santilli, Iso-Minkowskian Geometry for Interior Dynamical Problems, Contributed paper to Cosmology, Quantum Vacuum, and Zeta Functions, Diego Sez-Gmez Sergei Odintsov Sebasti Xamb Editors, Springer, 2011. http://www. santilli-foundation.org/docs/Isominkowskian-Geom.pdf.

[7] R.M. Santilli, Formulation, Interpretation and Verification of Isorelativity for Interior Dynamical Problems, contributed paper to the Proceedings of the San Marino Workshop on Astrophysics and Cosmology for Matter and Antimatter, Republic of San Marino, September 5 to 9, 2011, in press, 2012.

[8] R.M. Santilli, Experimental Verifications of IsoRedShift with Possible Absence of Universe Expansion, Big Bang, Dark Matter, and Dark Energy, The Open Astronomy Journal 3 (2010), 124. http://www.santilli-foundation.org/docs/Santilliisoredshift.pdf.

[9] R.M. Santilli, Experimental Verification of IsoRedShift and its Cosmological Implications, contributed paper to the Proceedings of the International Conference on Numerical Analysis and Applied Mathematics, Rhodes, Greece, September 19-25, 2010, T.E. Simos, ed., American Institute of Physics Conference Proceedings Vol. 1281, 2010, pp. 882-885. http://www.santilli-foundation.org/docs/Isoredshift-Letter.pdf.

[10] G. West and G. Amato, Experimental Confirmation of Santilli's IsoRedShift and IsoBlueShift, contributed paper to the Proceedings of the San Marino Workshop on Astrophysics and Cosmology for Matter and Antimatter, Republic of San Marino, September 5 to 9, 2011, in press, 2012.

[11] L.M. Lubin and A. Sandage, Astron J 122 (2001), 1084. http://arxiv.org/abs/astro-ph/0106566.

[12] N. Bloembergen, The stimulated Raman effect, Am J Phys 35 (1967), 989.

[13] Stanley David Gedzelman, Simulating colors of clear and partly cloudy skies, Applied Optics 44 (2005), 5723.

[14] M. Vollmer and S.D. Gedzelman, Colours of the Sun and Moon: the role of the optical air mass, Eur J Phys 27 (2006), 299.

[15] R. Verreault, Crystallographic, optical and magnetic properties of Eu2SiO4, Phys Kondens Materie 14 (1971), 37.

[16] G. Busch and R. Verreault, A new method to demonstrate and measure birefringence, Acta Cryst A 27 (1971), 680.

[17] R. Verreault, A new method to measure general birefringence in crystals, Z Kristallogr 136 (1972), 350.

[18] P. Atkins and S. de Paul, Physical Chemistry, Oxford Univ. Press, 7th ed., Section 16.3 (2002).

[19] R.M. Santilli, G. West and G. Amato, Documentation of IRS in the Sun, http://www.santilli-foundation.org/docs/IRSsun.zip.

[20] H. Arp. Quasars Redshift and Controversies. Interstellar Media, Berkeley, 1987.

[21] A.L. Kholmetskii et al., Experimentally observed deviations from the Mosbauer effect in rotating discs, contributed paper to the Proceedings of the San Marino Workshop on Astrophysics and Cosmology for Matter and Antimatter, Republic of San Marino, September 5 to 9, 2011, in press, 2012. 
[22] R. Verreault, Experimental confirmation of Santilli's prediction of the anisotropy of Earth's gravitational field via the spherical pendulum, contributed paper to the Proceedings of the San Marino Workshop on Astrophysics and Cosmology for Matter and Antimatter, Republic of San Marino, September 5 to 9, 2011, in press, 2012.

[23] G. Nimtz, Experimental evidence of superluminal communicvations, contributed paper to the Proceedings of the San Marino Workshop on Astrophysics and Cosmology for Matter and Antimatter, Republic of San Marino, September 5 to 9, 2011, in press, 2012.

[24] T. Adama et al., Measurement of the neutrino velocity with the OPERA detector in the CNGS beam, CERN preprint 2011, submitted for publication. http://www.santilli-foundation.org/docs/cern-paper.pdf.

[25] A. Bunthaler et al., III Zw2, The first superluminal jet in a Sayfert galaxy, A\&A 2000; 357, L45.

[26] R.M. Santilli and A.O.E. Animalu, Nonunitary Lie-isotopic and Lie-admissible scattering theories of hadronic mechanics, Papers I, II, III, IV and V, in the Proceedings of the Third International Conference on the Lie-Admissible Treatment of Irreversible Processes, C. Corda, ed., Kathmandu University, 2011, pp. 163-177. http://www.santilli-foundation.org/docs/Isoscattering-I.pdf; http://www.santilli-foundation.org/docs/Isoscattering-II.pdf; http://www.santilli-foundation.org/docs/Isoscattering-III.pdf; http://www.santilli-foundation.org/docs/Isoscattering-IV.pdf; http://www.santilli-foundation.org/docs/Isoscattering-V.pdf.

[27] E. Hubble, Proceedings of the National Academy of Sciences of the United States of America, 15 (1929), 168.

[28] F. Zwicky, On the Red Shift of Spectral Lines through Interstellar Space, Proceedings of the National Academy of Sciences of the United States of America 15 (1929), 773. http://www.pnas.org/cgi/reprintframed/15/10/773.

[29] C.W. Misner, K.S. Thorne and J.A. Wheeler, Gravitation, W.H. Feeman and Company, 1973.

[30] A. Landau and E. Lifsits, Classical Theory of Fields (3rd ed.), London: Pergamon, 1971.

[31] P. Riess et al., Astronomical Journal 116 1998), 1009; S. Perlmutter et al., Astrophysical Journal 517 (1999), 565.

[32] E.J. Wollack, Edward, The Study of the Universe: Big Bang Theory, NASA, 2010.

[33] A.B. Whiting, The expansion of space: free-particle motion and the cosmological redshift, The Observatory 124 (2004), 174. http://adsabs.harvard.edu/abs/2004Obs...124..174W.

[34] V. Rubin, Rotation of the Andromeda Nebula from a Spectroscopic Survey of Emission Regions, Astrophysical Journal 159 (1970), 379. http://adsabs.harvard.edu/doi/10.1086/150317.

[35] M. Everaldo de Souza, A Quantitative Model for Rotation of Spiral Galaxies without Dark Matter, contributed paper to the Proceedings of the San Marino Workshop on Astrophysics and Cosmology for Matter and Antimatter, Republic of San Marino, September 5 to 9, 2011, in press, 2012.

[36] P.J. Peebles and B. Ratra, The cosmological constant and dark energy, Reviews of Modern Physics 75 (2003, 559. http://adsabs.harvard.edu/abs/2003RvMP...75..559P.

[37] R.M. Santilli, The etherino and/or the Neutrino Hypothesis? Found Phys 37 (2007), 670. www.santilli-foundation.org/ docs/EtherinoFoundPhys.pdf.

[38] R.M. Santilli, Isodual Theory of Antimatter with Application to Antigravity, Grand Unification and the Spacetime Machine, Springer 2001. http://www.santilli-foundation.org/docs/santilli-79.pdf.

[39] R.M. Santilli, Can antimatter asteroids, stars and galaxies be detected with currtent means? in the Proceedings of the Third International Conference on the Lie-Admissible Treatment of Irreversible Processes, C. Corda, Editor, Kathmandu Universit p. 25 (2011). http://www.santilli-foundation.org/docs/Antimatter-Asteroid.pdf.

[40] R.M. Santilli, The mystery of detecting antimatter asteroids, stars and galaxies, Lecture 13 of http://www.world-lectureseries.org/san-marino-2011.

[41] I. Gandzha and J. Kadeisvili, New Sciences for a New Era: Mathematical, Physical and Chemical Discoveries of Ruggero Maria Santilli, Sankata Printing Press, Nepal (2011), http://www.santilli-foundation.org/docs/RMS.pdf. 